\title{
CARLO GASBARRI
}

\section{Hauteurs canoniques sur l'espace de modules des fibrés stables sur une courbe algébrique}

\author{
Bulletin de la S. M. F., tome 125, no 4 (1997), p. 457-491 \\ <http://www.numdam.org/item?id=BSMF_1997_125_4_457_0>
}

(C) Bulletin de la S. M. F., 1997, tous droits réservés.

L'accès aux archives de la revue «Bulletin de la S. M. F. » (http: //smf.emath.fr/Publications/Bulletin/Presentation.html) implique l'accord avec les conditions générales d'utilisation (http://www.numdam.org/ conditions). Toute utilisation commerciale ou impression systématique est constitutive d'une infraction pénale. Toute copie ou impression de ce fichier doit contenir la présente mention de copyright.

\section{Numdam}




\title{
HAUTEURS CANONIQUES SUR L'ESPACE DE MODULES DES FIBRÉS STABLES SUR UNE COURBE ALGÉBRIQUE
}

\author{
PAR CARLO GASBARRI (*)
}

RÉSUMÉ. - On construit une hauteur sur l'espace des fibrés stables de rang et de déterminant fixés sur une courbe sur un corps de nombres, dans le cas où le rang et le degré sont premiers entre eux et la courbe a partout une bonne réduction. Cette hauteur est définie en utilisant la théorie d'Arakelov

ABSTRACT. - We construct a canonical height on the moduli space of stable fibre bundles with fixed rank and determinant over an algebraic curve over a number field in the case when the degree and the rank are coprime and the curve has good reduction everywhere. This height is defined using Arakelov intersection theory.

\section{Introduction}

Soient $K$ un corps de nombres et $X_{K}$ une courbe lisse de genre $g \geq 1$ définie sur $K$. On sait que l'on peut définir une hauteur canonique $h_{\mathrm{NT}}$ sur la jacobienne de $X_{K}$ qu'on appelle hauteur de Néron-Tate.

Soit $\mathcal{O}_{K}$ l'anneau des entiers de $K$ et soit $X$ le modèle régulier minimal sur $\operatorname{Spec}\left(\mathcal{O}_{K}\right)$ de $X_{K}$; la théorie d'Arakelov est une théorie d'intersection entre les diviseurs «compactifiés » sur $X$.

On sait, d'après le théorème de Faltings-Hriljac, que, si $\mathcal{L}$ est un diviseur compactifié de degré zéro (qui intersecte tout diviseur vertical en degré zéro) alors

$$
h_{\mathrm{NT}}(\mathcal{L})=-\frac{1}{2[K: \mathbb{Q}]}(\mathcal{L} ; \mathcal{L}),
$$

où, sur la droite, on a l'intersection d'Arakelov (cf. [Fa], [Ga] ou [MB]).

(*) Texte reçu le 15 avril 1996, révisé le 4 juillet 1997, accepté le 20 mai 1997.

C. Gasbarri, Université de Rennes 1, Département de Mathématiques, Campus de Beaulieu, 35000 Rennes CEdEx (France).

Email : gasbarri@forestiere.univ-rennes1.fr

Classification AMS : 11G30, 14-xx, 14D20, 14G40.

Mots clés : Surfaces arithmétiques, fibrés vectoriels, espaces de modules, théorie d'Arakelov, hauteurs. 
Supposons que $f: X \rightarrow B=\operatorname{Spec}\left(\mathcal{O}_{K}\right)$ soit lisse, et supposons que $f$ possède une section $P$. Alors, à l'aide du faisceau inversible $\mathcal{O}_{X}(P)$ on peut identifier la jacobienne $J_{X / B}^{0}$ de $X$ sur $B$ à la composante $J_{X / B}^{d}$ du schéma de Picard de $X$ sur $B$ qui classifie les diviseurs de degré relatif $d$. Donc la hauteur de Néron-Tate définit une hauteur sur $J_{X / B}^{d}$ qu'on peut décrire en utilisant la théorie d'Arakelov.

Un outil pour l'étude de l'arithmétique des fibrés de rang plus grand que 1 sur $X_{K}$ pourrait être une hauteur canonique (analogue à la hauteur de Néron-Tate) sur l'espace de modules des fibrés semi-stables sur $X_{K}$.

Dans cet article on construit une telle hauteur dans certains cas.

On suppose toujours que $f: X \rightarrow B$ est un morphisme lisse et projectif et qu'il existe une section $P: B \rightarrow X$.

Soient $r>1$ un entier et $d$ un entier premier avec $r$. Soit $\mathcal{F}_{K}$ un fibré inversible sur $X_{K}$ tel que $\operatorname{deg}\left(\mathcal{F}_{K}\right)=d$.

Soit $\mathfrak{U}_{X_{K}}\left(r ; \mathcal{F}_{K}\right)$ l'espace de modules des fibrés sur $X_{K}$ stables de rang $r$ et de déterminant isomorphe à $\mathcal{F}_{K}$.

Dans cet article on construit une hauteur canonique sur $\mathfrak{U}_{X_{K}}\left(r ; \mathcal{F}_{K}\right)$ en utilisant la théorie d'Arakelov.

Soit $\mathcal{F}$ un modèle de $\mathcal{F}_{K}$ sur $X$ et soit $S(r ; \mathcal{F})$ la famille des classes d'isomorphisme des fibrés sur $X$ de rang $r$ et tels que leur restriction à chaque fibre de $f$ soit stable et la restriction du déterminant soit isomorphe à la restriction de $\mathcal{F}$.

Soit $E_{K}$ un fibré stable de rang $r$ et déterminant isomorphe à $\mathcal{F}_{K}$ sur $X_{K}$. Dans les paragraphes 3,4 et 5 , on verra comment on peut construire un fibré $\mathcal{E} \in S(r ; \mathcal{F})$ tel que $\mathcal{E} \otimes_{\mathcal{O}_{K}} K \simeq E_{K}$.

Pour tout $\sigma \in S_{\infty}$ on fixe une métrique d'Einstein-Hermite par rapport à la métrique d'Arakelov sur $\mathcal{E}_{\sigma}=\mathcal{E} \otimes_{\sigma} \mathbb{C}$ (pour les définitions voir après).

On dénote $\overline{\mathcal{E}}$ le fibré $\mathcal{E}$ muni de ces métriques. Soient $\widehat{c_{i}(\overline{\mathcal{E}})}$ les classes de Chern Arakeloviennes définies par exemple dans [D], dans [GS2] ou dans $[\mathrm{E}],\left(\widehat{c_{1}(\overline{\mathcal{E}})} \in \mathrm{Pic}_{c}(X)\right.$ et $\left.\widehat{c_{2}(\overline{\mathcal{E}})} \in \mathbb{R}\right)$. On considère enfin le nombre

$$
h\left(E_{K}\right)=\frac{1}{[K: \mathbb{Q}]}\left(2 r \widehat{c_{2}(\overline{\mathcal{E}})}-(r-1)\left(\widehat{c_{1}(\overline{\mathcal{E}})} ; \widehat{c_{1}(\overline{\mathcal{E}})}\right)\right) .
$$

On prouve que $h\left(E_{K}\right)$ dépend seulement de $E_{K}$ et pas des choix faits.

Le principal résultat de cet article est :

THÉORÈME. - Il existe un faisceau inversible ample $\Delta$ sur $\mathfrak{U}_{X_{K}}\left(r ; \mathcal{F}_{K}\right)$ tel que la fonction

$$
h(\cdot): \mathfrak{U}_{X_{K}}\left(r ; \mathcal{F}_{K}\right) \longrightarrow \mathbb{R}
$$

est une hauteur sur $\mathfrak{U}_{X_{K}}(r ; \mathcal{F})$ associée à $\Delta$.

TOME $125-1997-\mathrm{N}^{\circ} 4$ 
Remarque. - Moriwaki [Mo] et Soulé [So] ont prouvé que si $\mathcal{E}$ est stable sur la fibre générique de $f$, alors

$$
2 r \widehat{c_{2}(\mathcal{E})}-(r-1)\left(\widehat{c_{1}(\mathcal{E})} ; \widehat{c_{1}(\mathcal{E})}\right) \geq 0 .
$$

Cet article est organisé de la façon suivante.

On considère le foncteur $S_{X}(r ; d)(T)$ qui à chaque schéma $T$ sur $B$ lui associe l'ensemble des familles des fibrés sur $X$ semi-stables de rang $r$ et degré $d$ sur chaque fibre de $f$ paramétrisées par $T$ à l'action de $\operatorname{Pic}(T)$ près (pour une définition précise du foncteur, voir le paragraphe 3 ).

Dans la première partie de cet article (paragraphes 3 et 4), on démontre qu'il existe un schéma projectif sur $B$ qui classifie $S_{X}(r ; d)(\cdot)$; plus précisément, on démontre :

ThÉORÈmE 1. - Soient $r$ et $d$ deux entiers et $f: X \rightarrow B=\operatorname{Spec}\left(\mathcal{O}_{K}\right)$ une surface arithmétique (projective) à bonne réduction partout. Il existe un schéma projectif sur $B, g: \mathfrak{U}_{X}(r ; d) \rightarrow B$ qui est un espace de modules grossier pour le foncteur $S_{X}(r ; d)(\cdot)$.

Dans le cas où $r$ et $d$ son premiers entre eux, on construit une famille universelle sur $X \times_{B} \mathfrak{U}_{X}(r ; d)$ : on construit (paragraphe 5 ) un fibré $\mathcal{E}$ sur $X \times_{B} \mathfrak{U}_{X}(r ; d)$ qui vérifie la proposition suivante :

Proposition 2. - Soient $r$ et $d$ deux entiers premiers entre eux. Il existe un fibré de rang $r, \mathcal{E}$ sur $X \times_{B} \mathfrak{U}_{X}(r ; d)$ tel que, pour tout $B$ schéma $T$ et pour tout $P \in \mathfrak{U}_{X}(r ; d)(T)$, le fibré sur $X \times_{B} T$ obtenu par le diagramme cartésien

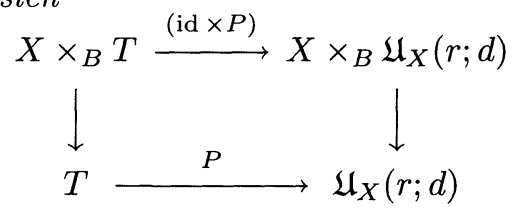

est dans $S_{X}(r ; d)(T)$. De plus si $\boldsymbol{E}$ est un élément de $S_{X}(r ; d)(T)$, de sorte que $\boldsymbol{E}$ définit un $B$-morphisme $\psi_{\boldsymbol{E}}: T \rightarrow \mathfrak{U}_{X}(r ; d)$, alors il existe $\mathcal{N} \in \operatorname{Pic}(T)$ tel que

$$
\left(\mathrm{id} \times \psi_{\boldsymbol{E}}\right)^{*}(\mathcal{E}) \simeq \boldsymbol{E} \otimes p_{2}^{*}(\mathcal{N})
$$

$\left(p_{2}: X_{T} \rightarrow T\right.$ étant la deuxième projection $)$.

Donc, si $r$ et $d$ sont deux entiers premiers entre eux, $\mathfrak{U}_{X}(r ; d)$ représente le foncteur $S_{X}(r ; d)(\cdot)$.

Pour toute place à l'infini $\sigma \in S_{\infty}$ de $K$, on munit (proposition 5.2) le fibré $\mathcal{E}_{\sigma}$ sur $\left(X \times_{B} \mathfrak{U}_{X}(r ; d)\right)_{\sigma}$ d'une métrique $C^{\infty}$ telle que, pour tout $q \in\left(\mathfrak{U}_{X}(r ; d)\right)_{\sigma}$ la métrique sur le fibré stable $\mathcal{E}_{\sigma \mid X_{\sigma} \times\{q\}}$ soit d'EinsteinHermite par rapport à la métrique d'Arakelov sur $X_{\sigma}$. 
De façon analogue on considère le foncteur $S_{X}(r ; \mathcal{F})(T)$ qui à chaque schéma $T$ sur $B$ lui associe l'ensemble des familles des fibrés sur $X$ semistables de rang $r$, de degré $d$ et de déterminant isomorphe à $\mathcal{F}$ sur chaque fibre de $f$ paramétrisées par $T$ à l'action de $\operatorname{Pic}(T)$ près (pour des définitions plus précises, regarder le $\S 6$ ).

Comme corollaire du théorème 1 et de la proposition 2 , on trouve :

THÉORÈme 3.- Si $r$ et $d$ sont premiers entre eux, il existe un B-schéma $\mathfrak{U}_{X}(r ; \mathcal{F})$ qui représente le foncteur $S_{X}(r ; \mathcal{F})$.

À la fin du sixième paragraphe, on construit la hauteur sur $\mathfrak{U}_{X_{K}}\left(r ; \mathcal{F}_{K}\right)$ l'espace de modules des fibrés semi-stables sur $X_{K}$ de rang $r$ et de déterminant isomorphe à $\mathcal{F}_{K}$, où $\mathcal{F}_{K}$ est un faisceau inversible de degré $d$ sur $X_{K}$, dans le cas où $r$ et $d$ sont premiers entre eux et le modèle minimal régulier de $X_{K}$ a une bonne réduction partout.

Remarque. - On utilisera ici la convention suivante : soit $T$ un $B$ schéma; un point géométrique de $T$ est un $B$-morphisme $\operatorname{Spec}(k) \rightarrow T$ où $k$ est un corps avec un morphisme (non constant) $\alpha: \mathcal{O}_{K} \rightarrow k$ tel que $k$ soit une extension séparable du corps des fractions de $\alpha\left(\mathcal{O}_{K}\right)$.

REMARQUe. - Le théorème 1 et la proposition 2 sont des cas particuliers des théorèmes prouvés dans [Ma] dans un cadre très général; on donne ici une démonstration ad hoc pour les surfaces arithmétiques qui est, évidemment, plus simple.

Je voudrais remercier J.B. Bost, L. Moret-Bailly, C. Soulé, S. Zhang et plus particulièrement L. Szpiro pour leur aide pendant l'écriture de cet article. Je voudrais aussi remercier le rapporteur pour ses remarques et ses corrections.

\section{Rappels et notation}

Soient $k$ un corps commutatif et $X$ une courbe lisse projective sur $k$. Soit $E$ un fibré sur $X$ de rang $r>0$ et degré $d$; on appelle pente de $E$ le nombre rationnel

$$
\mu(E)=\frac{d}{r}
$$

Plus généralement, on peut définir la pente de tout faisceau cohérent de rang positif de façon analogue; en effet on peut définir le rang et le degré de tout élément de $K_{0}(X)$.

TOME $125-1997-\mathrm{N}^{\circ} 4$ 
On dira que $E$ est semi-stable (stable) si pour tout sous faisceau $F \subset E$ (et $F \neq E$ ) on a

$$
\begin{cases}\mu(F) \leq \mu(E) & (\text { cas semi-stable }) \\ \mu(F)<\mu(E) & \text { (cas stable) }\end{cases}
$$

On sait que pour vérifier si $E$ est semi-stable (resp. stable) il suffit de vérifier (1) seulement pour les sous-fibrés.

Proposition 2.1. - Soient $E$ et $F$ deux fibrés semi-stables sur $X$. Si $\operatorname{Hom}(E ; F) \neq 0$, on $a$

$$
\mu(E) \leq \mu(F)
$$

et les seuls endomorphismes de E sont les homothéties.

Proposition 2.2. - Soit $E$ un fibré sur $X$; il existe une unique filtration

$$
\{0\} \subset F_{1} \subset F_{2} \subset \cdots \subset F_{k}=E
$$

telle que :

a) le fibré $\mathrm{gr}_{i}=F_{i} / F_{i-1}$ est semi-stable;

b) $\mu\left(\mathrm{gr}_{i}\right)>\mu\left(\mathrm{gr}_{i+1}\right)$.

La filtration est appelée filtration de Harder-Narasimhan de $E$ et $F_{1}$ est appelé sous-fibré déstabilisant maximal; il est évidemment semi-stable.

Proposition 2.3. - Soit $E$ un fibré semi-stable sur $X$ de rang $r$ et pente $\mu$; on $a$ :

$$
\frac{h^{0}(E)}{r} \leq \max (\mu+1 ; 0) .
$$

Pour des démonstrations, regarder [SD], [LP].

\section{Fibrés vectoriels stables sur une surface arithmétique}

Soient $K$ un corps de nombres et $\mathcal{O}_{K}$ son anneau d'entiers.

Soit $f: X \rightarrow B=\operatorname{Spec}\left(\mathcal{O}_{K}\right)$ une surface arithmétique lisse : à savoir, $f$ est un morphisme projectif lisse à fibres géométriquement connexes de dimension relative 1 (donc $f_{*}\left(\mathcal{O}_{X}\right) \simeq \mathcal{O}_{B}$ universellement). Soient $X_{K}$ la fibre générique de $f$ et $g=g\left(X_{K}\right)>0$ son genre. Supposons, de plus, qu'il existe une section $P: B \rightarrow X$.

Soient $r$ et $d$ deux entiers. Soit $\mathcal{E}$ un fibré sur $X$ de rang $r$ et de degré $d$; le degré de $\mathcal{E}$ est le degré de $\mathcal{E}_{K}$ sur la fibre générique $X_{K}\left(\mathcal{E}_{K}=\mathcal{E} \otimes_{\mathcal{O}_{K}} K\right)$.

BULLETIN DE LA SOCIÉTÉ MATHÉMATIQUE DE FRANCE 
Définition. - Soit $\mathcal{E}$ un fibré sur $X$.

a) On dira que $\mathcal{E}$ est génériquement semi-stable (génériquement stable) si $\mathcal{E}_{K}$ est un fibré semi-stable (stable) sur $X_{K}$.

b) On dira que $\mathcal{E}$ est semi-stable (stable) si pour tout $\mathfrak{p} \in B$, le fibré $\mathcal{E}_{\mathfrak{p}}$ est semi-stable (stable) sur $X_{\mathfrak{p}}$.

Remarque. - Si $\mathcal{E}$ est génériquement semi-stable, on peut prouver qu'il existe un ouvert non vide $U$ de $B$ tel que pour tout $\mathfrak{q} \in U$, le fibré $\mathcal{E}_{\mathfrak{q}}$ est semi-stable sur $X_{\mathfrak{q}}$ (la démonstration n'est pas élémentaire, $c f$. par exemple [LP2]).

Les deux propositions qui suivent seront constamment utilisées par la suite.

Proposition 3.1. - Soit $K^{\prime}$ une extension finie de $K$ et soit $\mathcal{O}_{K^{\prime}}$ son anneau d'entiers; soient $X^{\prime}=X \times_{B} \operatorname{Spec}\left(\mathcal{O}_{K^{\prime}}\right)$ et $\mathcal{E}^{\prime}=\operatorname{pr}_{X}^{*} \mathcal{E}\left(\operatorname{pr}_{X}\right.$ étant la projection naturelle de $X^{\prime}$ sur $\left.X\right)$. Alors $\mathcal{E}$ est semi-stable si et seulement si $\mathcal{E}^{\prime}$ est semi-stable.

Preuve. - Il suffit de prouver le lemme suivant :

Lemme 3.2. - Soient $k$ un corps fini et $k^{\prime}$ une extension de $k$. Soient $X$ une courbe lisse sur $k$ et $E$ un fibré sur $X$. Soient $X^{\prime}=X \times_{k} \operatorname{Spec}\left(k^{\prime}\right)$ et $E^{\prime}=\operatorname{pr}_{X}^{*} E\left(\right.$ où $\mathrm{pr}_{X}$ est la projection naturelle de $\left.X^{\prime} \grave{a} X\right)$. Alors $E$ est semi-stable si et seulement si $E^{\prime}$ est semi-stable.

Preuve. - Le revêtement $\mathrm{pr}_{X}: X^{\prime} \rightarrow X$ est galoisien. Supposons que $E$ ne soit pas semi-stable, alors évidemment $E^{\prime}$ n'est pas semi-stable.

Supposons que $E^{\prime}$ ne soit pas semi-stable; soit $F^{\prime}$ le sous faisceau déstabilisant maximal de $\mathcal{E}^{\prime}$; soit $\sigma \in \operatorname{Gal}\left(X^{\prime} / X\right)$. On a $\sigma^{*} E^{\prime}=E^{\prime}$ et donc, par unicité du sous faisceau déstabilisant maximal, $\sigma^{*} F^{\prime}=F^{\prime}$, d'où $F^{\prime}$ provient de $X$, donc $E$ n'est pas semi-stable.

Remarque. - En effet dans le lemme il suffit de supposer que $k$ est parfait.

La proposition 3.1, et surtout le lemme 3.2 sont à la base de beaucoup de considérations : elles impliquent que la semi-stabilité d'un fibré peut être lue sur la clôture algébrique de $K$. Donc, dans toute l'analyse de la stabilité on peut se réduire à des considérations de type géométrique (sur un corps algébriquement clos); évidemment non sans précautions.

Proposition 3.3. - Soit $h: T \rightarrow B$ un B-schéma. Soit $\mathcal{E}$ un fibré sur $X_{T}=X \times_{B} T$ tel que, pour tout point géométrique $t \in T$, le fibré $\mathcal{E}_{t}$ sur

TOME $125-1997-\mathrm{N}^{\circ} 4$ 
la courbe $X_{t}$ soit stable de rang $r$ et degré $d$. Alors

$$
\operatorname{pr}_{2 *}(\operatorname{End}(\mathcal{E})) \simeq \mathcal{O}_{T}
$$

$\mathrm{pr}_{2}: X_{T} \rightarrow T$ étant la deuxième projection.

Preuve. - Les seuls endomorphismes d'un fibré stable sur une courbe sur un corps algébriquement clos sont les homothéties. Si $t \in T$ est un point géométrique et $\overline{k(t)}$ est une clôture algébrique de $k(t)$, on a

$$
H^{0}\left(X_{t} \times_{k(t)} \overline{k(t)} ; \operatorname{End}\left(\mathcal{E}_{t}\right) \otimes \overline{k(t)}\right)=H^{0}\left(X_{t} ; \operatorname{End}\left(\mathcal{E}_{t}\right)\right) \otimes \overline{k(t)} .
$$

Cela implique que $\operatorname{dim}_{k(t)}\left(H^{0}\left(X_{t} ; \operatorname{End}\left(\mathcal{E}_{t}\right)\right)\right)=1$, pour tout point géométrique $t \in T$.

On a un morphisme injectif

$$
\begin{aligned}
\mathcal{O}_{T} & \longrightarrow \operatorname{End}(\mathcal{E}), \\
1 & \longmapsto \mathrm{id}
\end{aligned}
$$

où id est le morphisme identité.

On a donc trouvé une suite exacte de $\mathcal{O}_{T}$-modules

$$
0 \rightarrow \mathcal{O}_{T} \longrightarrow \operatorname{pr}_{2 *}(\operatorname{End}(\mathcal{E})) \text {. }
$$

Pour tout point $t \in T$, le morphisme composé

$$
\mathcal{O}_{T} \otimes k(t) \longrightarrow \operatorname{pr}_{2 *}(\operatorname{End}(\mathcal{E})) \otimes_{\mathcal{O}_{T}} k(t) \longrightarrow H^{0}\left(X_{t} ; \operatorname{End}\left(\mathcal{E}_{t}\right)\right)
$$

est un isomorphisme, donc $\mathcal{O}_{T} \simeq \operatorname{pr}_{2 *}(\operatorname{End}(\mathcal{E}))$ (cf. par exemple le cor. 2, p. 52 de $[\mathrm{Mu}])$.

Corollaire 3.4. - Soit $\mathcal{E}$ un fibré stable sur $X$; alors

$$
f_{*}(\operatorname{End}(\mathcal{E}))=\mathcal{O}_{B}
$$

Les propositions suivantes mettent en relation la géométrie d'un fibré (stable) sur $X$ avec la géométrie du fibré sur chaque fibre.

Proposition 3.5. - Soit $h: T \rightarrow B$ un B-schéma. Soient $\mathcal{E}$ et $\mathcal{F}$ deux fibrés sur $X_{T}=X \times_{B} T$. Supposons que pour tout point géométrique $t \in T$, les fibrés $\mathcal{E}_{t}$ et $\mathcal{F}_{t}$ sur la courbe $X_{t}$ soient stables de rang $r$ et de degré d. Supposons qu'il existe un recouvrement par ouverts $\mathcal{U}=\left\{U_{i}\right\}_{i \in I}$ de $T$ tel que, pour tout $i \in I$,

$$
\mathcal{E}_{\mid X \times U_{i}}=\mathcal{E}_{i} \simeq \mathcal{F}_{i}=\mathcal{F}_{\mid X \times U_{i}} .
$$

Alors il existe un fibré inversible $\mathcal{L}$ sur $T$ tel que

$$
\mathcal{E} \simeq \mathcal{F} \otimes \operatorname{pr}_{2 *}(\mathcal{L})
$$

où $\mathrm{pr}_{2}: X_{T} \rightarrow T$ est la projection canonique.

Preuve. - On a déjà remarqué que lorsque $E$ est un fibré stable sur une courbe $Y$, alors $h^{0}(Y ; \operatorname{End}(E))=1$. 
On a prouvé que $\operatorname{pr}_{2 *}\left(\operatorname{End}\left(\mathcal{E}_{i}\right)\right) \simeq \mathcal{O}_{U_{i}}$ sur $U_{i}$ (proposition 3.3). Par conséquent, le faisceau $\operatorname{pr}_{2 *}(\operatorname{Hom}(\mathcal{E} ; \mathcal{F}))$ est un fibré inversible sur $T$; notons-le $\mathcal{L}$. Donc $\operatorname{pr}_{2 *}\left(\operatorname{Hom}(\mathcal{E} ; \mathcal{F}) \otimes \operatorname{pr}_{2}^{*}\left(\mathcal{L}^{-1}\right)\right)$ est isomorphe à $\mathcal{O}_{T}$. Cela implique qu'il existe un élément $\operatorname{de} \operatorname{Hom}(\mathcal{E} ; \mathcal{F}) \otimes \operatorname{pr}_{2}^{*}\left(\mathcal{L}^{-1}\right)$ qui est non nul sur chaque fibre de $\mathrm{pr}_{2}$. Mais pour tout point géométrique $t \in T$, tout morphisme non nul $\left(\mathcal{E} \otimes \operatorname{pr}_{2}^{*}(\mathcal{L})\right)_{t} \rightarrow \mathcal{F}_{t}$ est un isomorphisme. Le lemme de Nakayama permet de conclure.

Corollaire 3.6. - Soient $\mathcal{E}$ et $\mathcal{F}$ deux fibrés stables sur $X$ tels que $\mathcal{E}_{K} \simeq \mathcal{F}_{K}$. Alors il existe $\mathcal{L} \in \operatorname{Pic}(B)$ tel que $\mathcal{E} \simeq \mathcal{F} \otimes f^{*} \mathcal{L}$.

Remarque. - Le corollaire n'est pas vrai, en général, si $\mathcal{E}$ (ou $\mathcal{F}$ ) n'est pas stable, mais il reste vrai si $f_{*}(\operatorname{End}(\mathcal{E}))$ est inversible.

REMARQUE. - Le corollaire 3.6 reste valable si $B$ est un schéma intègre.

Proposition 3.7. - Soit $\mathcal{F}$ un faisceau cohérent sur $X$ sans torsion tel que pour tout $\mathfrak{p} \in B$, le faisceau $\mathcal{F}_{\mathfrak{p}}$ est localement libre sur $X_{\mathfrak{p}}$. Alors $\mathcal{F}$ est localement libre sur $X$.

Preuve. - Soit $S(\mathcal{F})$ l'ensemble singulier de $\mathcal{F}$. Comme on a l'inégalité $\operatorname{codim}_{X}(S(\mathcal{F})) \geq 2$, on peut donc supposer que $B=\operatorname{Spec}(R)$ où $R$ est un anneau de valuation discrète; notons $K$ son corps des fractions et $k$ son corps résiduel.

Soit $s_{P}: B \rightarrow X$ une section de $f$; alors $s_{P}^{*} \mathcal{F}$ est un faisceau cohérent sur $B$ tel que $\operatorname{dim}_{K} s_{P}^{*} \mathcal{F} \otimes K=\operatorname{dim}_{k} s_{P}^{*} \mathcal{F} \otimes k$. Cela implique que $s_{P}^{*} \mathcal{F}$ est libre sur $B$. Il existe donc un voisinage de $s_{P}(B)$ dans $X$ où $\mathcal{F}$ est libre.

Puisque $X$ est plat sur $B$, les anneaux locaux des points (fermés ou pas) n'ont pas de $R$-torsion.

On voit aisément que les deux remarques ci-dessus impliquent que la fibre de $\mathcal{F}$ sur chaque section de $f$ de $X$ est un module libre sur l'anneau local de la section; donc il existe un voisinage de la section où $\mathcal{F}$ est localement libre.

Puisque $X$ est quasi-compacte, on peut trouver un recouvrement fini $B^{\prime} \rightarrow B$ (où $B^{\prime}=\operatorname{Spec}\left(R^{\prime}\right)$ et $R^{\prime}$ est un anneau principal) et un recouvrement fini $\mathcal{U}$ de $X^{\prime}=X \times_{B} B^{\prime}$ tels que pr* $\mathcal{F}$ est libre sur chaque ouvert de $\mathcal{U}$ (pr : $X^{\prime} \rightarrow X$ étant la projection naturelle). Donc $\operatorname{pr}^{*} \mathcal{F}$ est localement libre sur $X^{\prime}$.

Soit $\mathcal{F}^{* *}$ le bidual de $\mathcal{F}$. Le faisceau $\mathcal{F}^{* *}$ est localement libre sur $X$ car $X$ est régulier de dimension 2. Le morphisme canonique $\mathcal{F} \rightarrow \mathcal{F}^{* *}$ devient un isomorphisme quand on lui applique le foncteur $\operatorname{pr}^{*}(\cdot)$. Mais pr : $X^{\prime} \rightarrow X$ est fidèlement plat, donc $\mathcal{F}$ est isomorphe à son bidual et donc il est localement libre.

TOME $125-1997-\mathrm{N}^{\circ} 4$ 
Soient $r$ et $d$ deux nombres entiers. On veut étudier l'ensemble

$$
S_{X}(r ; d)=\{\mathcal{E} \mid \mathcal{E} \text { est un fibré stable sur } X
$$

de rang $r$ et de degré $d\} / \sim$

où $\mathcal{E} \sim \mathcal{E}^{\prime}$ si $\mathcal{E}$ est isomorphe à $\mathcal{E}^{\prime}$.

On voit aisément que, si $\mathcal{L}$ est un faisceau inversible de degré $n$ sur $X$, il existe une application bijective

$$
\begin{aligned}
S_{X}(r ; d) & \longleftrightarrow S_{X}(r ; d+r n), \\
{[\mathcal{E}] } & \longleftrightarrow[\mathcal{E} \otimes \mathcal{L}] .
\end{aligned}
$$

Donc, pour étudier $S_{X}(r ; d)$, on peut supposer que le degré est aussi grand que l'on veut.

Soit $\mathfrak{S}$ un ensemble de classes d'isomorphisme de faisceaux cohérents sur $X$. On rappelle qu'on dit que $\mathfrak{S}$ est une famille limitée modulo $\operatorname{Pic}(B)$ s'il existe un schéma $S \rightarrow B$ de type fini et un faisceau $\mathcal{F}$ sur $S \times_{B} X$ tels que pour tout $\mathcal{G} \in \mathfrak{S}$, il existe $s \in S(B)$ et $\mathcal{L} \in \operatorname{Pic}(B)$ tels que $\mathcal{G} \simeq \mathcal{F}(s) \otimes f^{*} \mathcal{L}\left(\right.$ où $\left.\mathcal{F}(s)=(s \times \text { id })^{*} \mathcal{F}\right)$.

On rappelle ( $c f .[\mathrm{M}])$ que, si $E$ est un fibré vectoriel de $\operatorname{rang} r \operatorname{sur} B$, alors $E$ est de la forme $\mathcal{O}_{B}^{r-1} \oplus \mathcal{L}$ avec $\mathcal{L} \in \operatorname{Pic}(B)$. En particulier, on a $K_{0}(B)=\operatorname{Pic}(B) \oplus \mathbb{Z}$ et il y a au plus $h=\operatorname{cl}\left(\mathcal{O}_{K}\right)=\operatorname{Card}(\operatorname{Pic}(B))$ classes d'isomorphisme de fibrés vectoriels de rang $r$ sur $B$.

Proposition 3.8. - L'ensemble $S_{X}(r ; d)$ est une famille limitée modulo $\operatorname{Pic}(B)$.

Preuve. - Soit $P \in X(B)$ la section qu'on a fixée au début de ce paragraphe et soit $\mathcal{O}_{X}(P)$ le faisceau inversible associé à $P$.

Soit $\mu=\frac{d}{r}$. Soit $\mathfrak{p} \in B$ et soit $k_{0}$ un entier tel que $k_{0}>2 g-1-\mu$. Soit $\mathcal{E} \in S_{X}(r ; d) ;$ alors $\operatorname{Hom}\left(\left(\mathcal{E} \otimes \mathcal{O}_{X}\left(k_{0} P\right)\right)_{\mathfrak{p}} ; \omega_{X \mathfrak{p}}\right)=0$ (proposition 2.1 ) donc, par dualité de Serre,

$$
H^{1}\left(X_{\mathfrak{p}} ;\left(\mathcal{E} \otimes \mathcal{O}_{X}\left(k_{0} P\right)\right)_{\mathfrak{p}}\right)=0
$$

Cela entraîne que $R^{1} f_{*}\left(\mathcal{E} \otimes \mathcal{O}_{X}\left(k_{0} P\right)\right)=0$ et donc $f_{*}\left(\mathcal{E} \otimes \mathcal{O}_{X}\left(k_{0} P\right)\right)$ est un faisceau localement libre sur $B$ de rang $\chi=d+r k_{0}+r(1-g)$.

De plus, pour tout $\mathfrak{p} \in B$, le faisceau $\left.\mathcal{E} \otimes \mathcal{O}_{X}\left(k_{0} P\right)\right)_{\mathfrak{p}}$ est engendré par ses sections globales sur $X_{\mathfrak{p}}$.

On a donc un morphisme surjectif

$$
f^{*} f_{*}\left(\mathcal{E} \otimes \mathcal{O}_{X}\left(k_{0} P\right)\right) \otimes \mathcal{O}_{X}\left(-k_{0} P\right) \longrightarrow \mathcal{E} \rightarrow 0 .
$$


Le fibré sur $B, f_{*}\left(\mathcal{E} \otimes \mathcal{O}_{X}\left(k_{0} P\right)\right)$ est de la forme $\mathcal{O}_{K}^{\chi-1} \oplus \mathcal{L}$ avec $\mathcal{L} \in \operatorname{Pic}\left(\mathcal{O}_{K}\right)$.

Soit $\mathbb{Q}(\mathcal{L})$ le $B$-schéma

$$
\underline{\operatorname{Quot}}_{X / B}^{P(x)}\left(f^{*}\left(\mathcal{O}_{K}^{\chi-1} \oplus \mathcal{L}\right) \otimes \mathcal{O}_{X}\left(k_{0} P\right)\right)
$$

où $P(x)$ est le polynôme

$$
P(n)=\chi\left(\mathcal{G}_{\mathfrak{p}}(n)\right)=\sum_{i=1}^{\infty}(-1)^{i} \operatorname{dim}_{k_{\mathfrak{p}}} H^{i}\left(X_{\mathfrak{p}} ;\left(\mathcal{G} \otimes \mathcal{O}_{X}(n P)\right)_{\mathfrak{p}}\right)
$$

avec $\mathfrak{p}$ n'importe quel point de $B$ et $\mathcal{G}$ un élément de $S_{X}(r ; d)$.

Soit $\mathcal{F}$ la famille universelle $\operatorname{sur} \mathbb{Q}(\mathcal{L}) \times_{B} X$. On voit alors que pour tout $\mathcal{E} \in S_{X}(r ; d)$ tel que $f_{*}\left(\mathcal{E} \otimes \mathcal{O}_{X}\left(k_{0} P\right)\right) \simeq \mathcal{O}_{K}^{\chi-1} \oplus \mathcal{L}$, il existe un point $s \in \mathbb{Q}(\mathcal{L})(B)$ et un $\mathcal{M} \in \operatorname{Pic}(B)$ (grâce à la proposition 3.4 et la remarque après) tels que $\mathcal{F}(s) \otimes f^{*} \mathcal{M} \simeq \mathcal{E}$. Donc $S_{X}(r ; d)$ est une famille limitée modulo $\operatorname{Pic}(B)$.

\section{L'espace de modules des fibrés stables sur une surface arithmétique}

a) Géométrie invariante sur $\mathcal{O}_{K}$.

Pour les démonstrations des proposition de cette première partie, regar$\operatorname{der}[\mathrm{S}]$.

Soient $G$ un schéma en groupes réductifs sur $\mathcal{O}_{K}$ et $V$ un $\mathcal{O}_{K}$-module localement libre de rang fini. Soient $\rho$ une action linéaire de $G$ sur

$$
\mathbb{A}_{B}(V)=\operatorname{Spec}\left(\operatorname{Sym}\left(V^{*}\right)\right)
$$

et $X$ un sous-schéma fermé $G$-invariant de $\mathbb{A}_{B}(V)$.

DÉfinition. - Un point géométrique $x \in X(k)$ (où $k$ est un corps algébriquement clos avec un morphisme $\mathcal{O}_{K} \rightarrow k$ ) est dit semi-stable si la clôture (dans $X \otimes k$ ) de la $G \otimes k$ orbite de $x$ ne contient pas l'origine ( 0 ). Le point géométrique est stable si la $G \otimes k$ orbite de $x$ est fermée et sa dimension est la même que celle de $G \otimes k$.

Remarque. - Si $\operatorname{dim}(G \otimes k) \geq 1$ et si $x$ est stable, alors $x$ est semistable.

À l'aide de ces définitions on peut traiter le cas projectif.

Soit $\mathbb{P}_{B}(V)=\operatorname{Proj}\left(\operatorname{Sym}\left(V^{*}\right)\right)$; l'action de $G$ sur $V$ induit une action de $G$ sur $\mathbb{P}_{B}(V)$. Soit $Y$ un sous-schéma fermé de $\mathbb{P}_{B}(V)$ stable sous l'action de $G$; on pose $Y=\operatorname{Proj}(A)$ où $A$ est une $\mathcal{O}_{K}$-algèbre graduée quotient de $\operatorname{Sym}\left(V^{*}\right)$; soit $\widehat{Y}$ le cône au dessus de $Y$, i.e. $\widehat{X}=\operatorname{Spec}(A)$. On a une action canonique de $G$ sur $\widehat{Y}$.

TOME $125-1997-\mathrm{N}^{\circ} 4$ 
DÉfinition. - Un point géométrique $x \in Y(k)$ est semi-stable (stable) si pour un $\hat{x} \in \widehat{Y}(k)-(0)((0)$ est le sommet de $\widehat{Y}(k))$ au-dessus de $x, \hat{x}$ est semi-stable (stable) pour l'action de $G$ sur le schéma affine $\widehat{Y}$.

Proposition 4.1. - Soient $G, V$ et $Y$ définis comme précédemment :

a) Il existe un ouvert $Y^{\text {ss }}$ de $Y$ tel que les points géométriques de $Y^{\text {ss }}$ sont exactement les point semi-stables de $Y$. De plus, si on a un morphisme $T \rightarrow B$, on a

$$
\left(Y \times_{B} T\right)^{s s}=Y^{s s} \times_{B} T
$$

où, à gauche, on a le sous-schéma des points semi-stables pour l'action $d u$ $T$-schéma en groupes réductifs $G \times_{B} T$ sur le schéma projectif $Y \times_{B} T$.

b) $S i x_{1}, \ldots, x_{n}$ sont des points (fermés) semi-stables de $Y$, alors il existe un polynôme $F \in \operatorname{Sym}\left(V^{*}\right), G$-invariant, de degré positif tel que $F\left(x_{i}\right) \neq 0$.

c) Il existe un ouvert $Y^{s} \subset Y^{s s} \subset Y$ tel que, pour tout corps algébriquement clos $k$ (avec un morphisme non trivial $\mathcal{O}_{K} \rightarrow k$ ), l'ensemble $Y^{s}(k)$ est l'ensemble des points stables de $Y(k)$.

Quand on a un $\mathcal{O}_{K^{-}}$-schéma en groupes réductifs qui agit sur un $\mathcal{O}_{K^{-}}$ schéma projectif, on peut regarder «l'espace topologique quotient»:

Proposition 4.2. - Soient $G$ un $\mathcal{O}_{K}$-schéma en groupes réductifs et $V$ un $G-\mathcal{O}_{K}$-module localement libre de rang $n$. Soit $Y$ un sous-schéma fermé $G$-stable de $\mathbb{P}_{B}(V)$. Donc $Y=\operatorname{Proj}(A)$, pour une $\theta_{K}$-algèbre $\Delta$. Soient $Z=\operatorname{Proj}\left(A^{G}\right)$ (éléments invariants par l'action de $G$ ) et $\varphi^{\prime}$ l'application rationnelle donnée par l'inclusion $A^{G} \subset A$ :

$$
\varphi^{\prime}: Y-\cdots \rightarrow Z \text {. }
$$

Soient $Y^{\text {ss }}$ le sous-schéma ouvert $(G$-stable $)$ de $Y$ des points semistables de $Y$ et $\varphi=\left.\varphi^{\prime}\right|^{\text {ss }} ;$ alors :

1) $\varphi$ est un morphisme

$$
\varphi: Y^{s s} \longrightarrow Z \text {. }
$$

2) $\varphi$ est un morphisme $G$-invariant affine et $\mathcal{O}_{Z}=\varphi_{*}\left(\mathcal{O}_{Y^{s s}}\right)^{G}$.

3) $\varphi$ est surjectif : pour tout corps algébriquement clos $k\left(\right.$ avec $\left.\mathcal{O}_{K} \rightarrow k\right)$, on a l'identification

$$
Z(k)=Y^{s s}(k) / \sim
$$

où $x_{1} \sim x_{2}$ si $\overline{O\left(x_{1}\right)} \cap \overline{O\left(x_{2}\right)} \neq \emptyset$ (les adhérences sont les clôtures des orbites dans $\left.Y^{s s} \times_{\mathcal{O}_{K}} \operatorname{Spec}(k)\right)$. 
4) Pour tout sous-schéma fermé $G$-stable $F$ de $Y^{s s}, \varphi(F)$ est fermé dans $Z$; et si $F_{1}$ et $F_{2}$ sont deux sous-schémas fermés $G$-stables de $Y^{\text {ss }}$ tels que $F_{1} \cap F_{2}=\emptyset$, alors $\varphi\left(F_{1}\right) \cap \varphi\left(F_{2}\right)=\emptyset$.

5) Il existe un ouvert $Z^{s} \subset Z$ tel que $Y^{s}=\varphi^{-1}\left(Z^{s}\right)$ et pour tout corps algebriquement clôs $k$ (avec un morphisme $\mathcal{O}_{K} \rightarrow k$ ) on ait

$$
Z^{s}(k)=Y^{s}(k) / G(k) \text {. }
$$

6) Le schéma $Z$ est projectif sur $B$.

Pour une démonstration, regarder [S].

On peut donc appeler $Z$ le «schéma quotient de $Y$ sous l'action de $G$ » et on peut le noter $Z=Y / G$.

REMARQUE. - Quelques observations à propos de ces propositions sont nécessaires (et utilisés après) :

a) On peut prendre, à la place de $\mathcal{O}_{K}$, n'importe quel anneau $R \subset K$.

b) Le point 3) implique que pour tout point $\mathfrak{p} \in \operatorname{Spec}\left(\mathcal{O}_{K}\right)$, la fibre $Z_{\mathfrak{p}}$ de $Z$ au-dessus de $\mathfrak{p}$ est un bon quotient de $Y_{\mathfrak{p}}^{s s}$ sous l'action de $G_{\mathfrak{p}}$, donc il est un quotient catégorique de $Y_{\mathfrak{p}}^{s s}$. Si l'on sait qu'il existe un quotient géométrique de $Y_{\mathfrak{p}}^{s s}$ sous l'action de $G_{\mathfrak{p}}$, ce dernier est donc canoniquement isomorphe à $Z_{\mathfrak{p}}$ (par unicité du quotient catégorique).

c) Si $G$ agit sur un $B$-schéma, un bon quotient de $Y$ est un couple $(Z ; f)$ où $Z$ est un $B$-chéma et $f: Y \rightarrow Z$ est un $B$-morphisme qui vérifie les propriétés 2 ), 3) et 4 ) de la proposition 4.2 (avec $Y$ à la place de $Y^{s s}$. Pour plus de détails sur la définition de bon quotient, de quotient catégorique, etc., regarder aussi [SD, p. 29 et ss].

d) On peut prouver $(c f$. $[\mathrm{S}])$ que $Z$ est un quotient uniformément catégorique de $Y$ (i.e. commute aux changements de base plats, cf. [GIT, p. 4]).

b) La construction de l'espace de modules

Soient $R$ un anneau de Dedekind avec corps de fractions $K$ (le corps des nombres) et $f: X \rightarrow \operatorname{Spec}(R)=B_{R}$ une courbe lisse projective de genre $g>0$ munie d'une section $P \in X\left(B_{R}\right)$; soit $\mathcal{O}_{X}(P)$ le faisceau inversible sur $X$ associé à $P$.

Soit $\mathrm{Sch} / B_{R}$ la catégorie où les objets sont les schémas séparés sur $R$, et les morphismes sont les morphismes de $B_{R}$-schémas. Soient $T$ un $B_{R^{-}}$ schéma séparé $\left(T \in \mathrm{Ob}\left(\mathrm{Sch} / B_{R}\right)\right.$ et $\mathcal{E}$ un fibré sur $X_{T}=X \times_{B_{R}} T$.

On dira que $\mathcal{E}$ vérifie la propriété $\left(^{*}\right)$ si, pour tout point géométrique $t \in T$, le fibré $\mathcal{E} \otimes k(t)$ est un fibré semi-stable de rang $r$ et degré $d$ sur la courbe $X_{t}$, la fibre de $\mathrm{pr}_{2}$ au-dessus de $t\left(\mathrm{pr}_{2}: X_{T} \rightarrow T\right.$ étant la deuxième projection).

$$
\text { TOME } 125-1997-\mathrm{N}^{\circ} 4
$$


Soit

$$
S_{X}(r ; d)(\cdot): \operatorname{Sch} / B_{R} \longrightarrow \mathcal{E} n s
$$

le foncteur contravariant qui associe à $T \in \mathrm{Ob}\left(\mathrm{Sch} / B_{R}\right)$ l'ensemble

$$
\begin{array}{r}
S_{X}(r ; d)(T)=\left\{\mathcal{F} \mid \mathcal{F} \text { est un fibré sur } X \times{ }_{B_{R}} T=X_{T}\right. \\
\text { qui vérifie la propriété } \left.\left({ }^{*}\right)\right\} / \sim
\end{array}
$$

avec $\mathcal{F} \sim \mathcal{F}^{\prime}$ si $\mathcal{F} \simeq \mathcal{F}^{\prime} \otimes \operatorname{pr}_{2}^{*}(\mathcal{L})$, où $\mathcal{L}$ est un fibré inversible sur $T$ et $\mathrm{pr}_{2}: X_{T} \rightarrow T$ est la projection canonique.

Si $h: T^{\prime} \rightarrow T$ est un $B_{R}$-morphisme, alors, grâce au lemme 3.2 , on a un morphisme $(\mathrm{id} \times h)^{*}: S_{X}(r ; d)(T) \rightarrow S_{X}(r ; d)\left(T^{\prime}\right)$; donc $S_{X}(r ; d)(\cdot)$ est bien un foncteur.

Si $k$ est un corps algébriquement clos (avec un morphisme non trivial $\left.\mathcal{O}_{K} \rightarrow k\right)$, on note $\operatorname{St}_{X}(r ; d)$ l'ensemble des fibrés stables de rang $r$ et degré $d$ sur $X_{k}=X \times{ }_{B} \operatorname{Spec}(k)$.

On se propose ici d'étudier la représentabilité de ce foncteur. On remarque d'abord que, pour tout $n \in \mathbb{Z}$,

$$
S_{X}(r ; d)(\cdot) \simeq S_{X}(r ; d+r n)(\cdot) ;
$$

donc on peut supposer que le degré $d$ est aussi grand que l'on veut.

On arrivera seulement à construire un espace de modules grossier pour le foncteur $S_{X}(r ; d)(\cdot)$.

Dans le cas où $R$ est un corps, la construction de l'espace de modules des fibrés semi-stables de rang et de degré fixés est classique ( $c f$. par exemple $[\mathrm{SD}])$ : on sait qu'il existe un espace des modules grossier qu'en général on désigne par $\mathfrak{U}_{X}(r ; d)$; si $r$ et $d$ sont premiers entre eux, $\mathfrak{U}_{X}(r ; d)$ représente le foncteur $S_{X}(r ; d)(\cdot)$ (il est un espace des modules fin).

Toujours dans le cas où $R$ est un corps, on sait aussi que $\mathfrak{U}_{X}(r ; d)$ est réduit et irréductible de dimension $r^{2}(g-1)+1$; de plus, si $r$ et $d$ sont premiers entre eux, c'est une variété lisse

On suppose, pour le moment, que le groupe de Picard de $R$ est trivial ( $R$ est un anneau principal).

L'énoncé qui suit est à la base de notre construction.

ThÉORÈme 4.3. - Soient $r$ et $d$ deux entiers $(r>0)$; alors il existe un schéma $g: \mathfrak{U}_{X}(r ; d) \rightarrow B_{R}$, où $g$ est un morphisme projectif, et un morphisme de foncteurs

$$
\psi: S_{X}(r ; d)(\cdot) \longrightarrow \operatorname{Hom}_{B_{R}}\left(\cdot ; \mathfrak{U}_{X}(r ; d)\right)
$$

universel au sens suivant : pour tout $B_{R}$-schéma $N$ et tout morphisme fonctoriel

$$
\varphi: S_{X}(r ; d)(\cdot) \longrightarrow \operatorname{Hom}_{B_{R}}(\cdot ; N)
$$




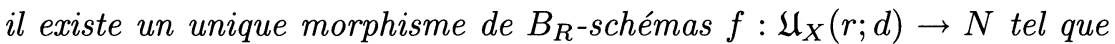
le diagramme suivant est commutatif

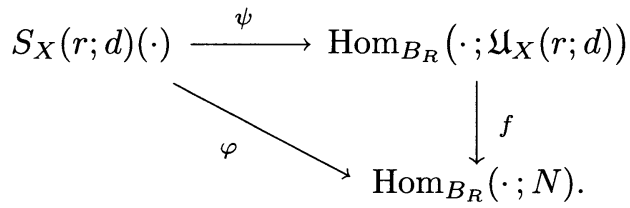

De plus, il existe un ouvert dense $U_{\text {st }} \subset \mathfrak{U}_{X}(r ; d)$ tel que pour tout corps algébriquement clos $k$ (avec un morphisme non trivial $R \rightarrow k$ ), on ait $U_{s t}(k)=S t_{X}(r ; d)(k)$. Ou, de façon plus syntétique, $\mathfrak{U}_{X}(r ; d)$ est un espace de modules grossier pour le foncteur $S_{X}(r ; d)(\cdot)$.

La démonstration de ce théorème est assez longue : elle est inspirée par la géométrie invariante sur $\mathcal{O}_{K}$ et par la construction classique de l'espace des modules des fibrés semi-stables sur une courbe sur un corps algébriquement clos ( $c f$. par exemple [LP] ou [SD]).

REMARQUE. - On peut trouver une autre démonstration de ce théorème dans [Ma, II], dans un cadre beaucoup plus général. La construction de l'espace de modules des fibrés semi-stables sur une surface arithmétique, qu'on donne ici est plus simple et plus explicite. On a ainsi l'avantage de mieux comprendre la géométrie et l'arithmétique d'un tel espace. De plus on peut se passer des nombreux problèmes techniques qu'on a dans des cadres plus généraux (dont la solution est souvent très longue et difficile à comprendre).

Preuve. - On commence par un lemme qui traduit la notion de stabilité en terme du foncteur $f_{*}(\cdot)$.

Lemme 4.4. - Soit $\operatorname{Coh}(r ; d)$ la famille des faisceaux cohérents sur $X$ de degré générique $d$ et de rang générique $r$. On peut trouver un entier $N(r ; d)$ (qui dépend seulement de $r, d$ et $g=g\left(X_{K}\right)$ ) tel que pour tout $n \geq N(r ; d)$ et $\boldsymbol{E} \in \operatorname{Coh}(r ; d)$ sans torsion, on ait l'équivalence :

1) $\boldsymbol{E}$ est localement libre et semi-stable;

2) pour tout $\mathfrak{p} \in B$ et $F_{\mathfrak{p}}$ sous-faisceau cohérent de rang $r^{\prime}$ (sur $\left.X_{\mathfrak{p}}\right)$ de $\boldsymbol{E}_{\mathfrak{p}}$, on $a$ :

$$
\operatorname{dim}_{k_{\mathfrak{p}}}\left(H^{0}\left(X_{\mathfrak{p}} ; F_{\mathfrak{p}} \otimes \mathcal{O}_{X}(n P)\right)\right) \leq \frac{r^{\prime}}{r} \operatorname{dim}_{k_{\mathfrak{p}}}\left(H^{0}\left(X_{\mathfrak{p}} ;\left(\boldsymbol{E} \otimes \mathcal{O}_{X}(n P)\right)_{\mathfrak{p}}\right) .\right.
$$

Preuve. - La démonstration est analogue à la démonstration de la prop. 7.1, p. 102 dans [LP]. On commence par une remarque. 
Soient $Y$ une courbe lisse de genre $g$ sur un corps $k, P \in Y(k), r$ et $d$ deux entiers et $\alpha$ un nombre positif. Soit $S_{\alpha}(r ; d)$ l'ensemble des classes d'isomorphisme des fibrés vectoriels $F$ sur $Y$ de rang $r$ et degré $d$ tels que pour tout $F^{\prime} \subset F$ on a $\mu\left(F^{\prime}\right) \leq \mu(F)+\alpha$; alors pour tout $F \in S_{\alpha}(r ; d)$ et $n \geq n_{0}=2 g-1+(r-1) \alpha-\mu(F)$, on a :

$$
H^{1}\left(X ; F \otimes \mathcal{O}_{Y}(n P)\right)=\{0\} .
$$

En effet, si $H^{1}\left(Y ; F \otimes \mathcal{O}_{Y}(n P)\right) \neq 0$, alors $\operatorname{Hom}\left(F \otimes \mathcal{O}_{Y}(n P) ; \omega_{Y}\right) \neq 0$, par dualité de Serre. Cela implique qu'il existe un fibré inversible de degré plus petit que $(2 g-2)$, quotient de $F \otimes \mathcal{O}_{Y}(n P)$; mais, par hypothèse, $2 g-2 \leq \mu(F)+n-(r-1) \alpha$ : on a donc une contradiction.

On remarque que $n_{0}$ ne dépend que de $r, d, \alpha$ et $g$.

Soient donc $\mathcal{E}$ un fibré semi-stable de rang $r$ et degré $d$ sur $X$ et $\mu=d / r$ sa pente. Soient $\mathfrak{p} \in B$ et $F_{\mathfrak{p}}$ un sous faisceau cohérent de $\mathcal{E}_{\mathfrak{p}}$ sur $X_{\mathfrak{p}}$; on peut supposer que $F_{\mathfrak{p}}$ et $\mathcal{E}_{\mathfrak{p}} / F_{\mathfrak{p}}$ sont localement libres sur $X_{\mathfrak{p}}$. Notons $r^{\prime}$ le rang de $F_{\mathfrak{p}}, \mu_{i}$ et $r_{i}$ les suites des pentes et des rangs de la filtration de Harder-Narasimhan de $F_{\mathfrak{p}}$ pour $i=1, \ldots, k_{0}$.

On pose $\nu=\mu_{k_{0}}$ et $[x]_{+}=\max (x ; 0)$. On a alors :

$$
\begin{aligned}
\frac{1}{r^{\prime}} h^{0}\left(X_{\mathfrak{p}} ; F_{\mathfrak{p}} \otimes \mathcal{O}_{X}(n P)\right) & \leq \sum_{i} \frac{r_{i}}{r^{\prime}}\left[\mu_{i}+n+1\right]_{+} \\
& \leq\left(1-\frac{1}{r}\right)[\mu+n+1]_{+}+\frac{1}{r}[\nu+n+1]_{+} .
\end{aligned}
$$

Soient $a=\mu-g r-1$ et $n \geq N^{\prime}(r ; d)=\max (-(a+1) ; 2 g-2-\mu)$; alors, si $\nu \leq a$, on trouve :

$$
\frac{1}{r^{\prime}} h^{0}\left(X_{\mathfrak{p}} ; F_{\mathfrak{p}} \otimes \mathcal{O}_{X}(n P)\right) \leq \mu+n+1-g .
$$

Mais $R^{1} f_{*}\left(\mathcal{E} \otimes \mathcal{O}_{X}(n P)\right)=0$, donc

$$
\frac{1}{r} h^{0}\left(X_{\mathfrak{p}} ;\left(\mathcal{E} \otimes \mathcal{O}_{X}(n P)\right)_{\mathfrak{p}}\right)=n+\mu+1-g .
$$

Supposons maintenant que $\nu \geq a$. Cela implique que $\mu\left(F_{\mathfrak{p}}\right)>a$; mais pour tout sous-faisceau $F_{\mathfrak{p}}^{\prime}$ de $F_{\mathfrak{p}}$, on a $\mu\left(F_{\mathfrak{p}}^{\prime}\right) \leq \mu$ (à cause de la stabilité de $\mathcal{E}$ ), d'où

$$
\mu\left(F_{\mathfrak{p}}^{\prime}\right) \leq \mu\left(F_{\mathfrak{p}}\right)+g r+1 .
$$

La condition $\nu>a$ et la remarque impliquent alors qu'il existe un entier $N_{0}=N_{0}(r ; d ; g)$ qui dépend seulement de $r, d$ et $g$ tel que pour $n \geq N_{0}$, on a $h^{1}\left(X_{\mathfrak{p}} ; F_{\mathfrak{p}}\left(\otimes \mathcal{O}_{X}(n P)\right)=0\right.$ et donc

$$
\frac{1}{r^{\prime}} \chi\left(X_{\mathfrak{p}} ; F_{\mathfrak{p}}\left(\otimes \mathcal{O}_{X}(n P)\right)=\frac{1}{r} h^{0}\left(X_{\mathfrak{p}} ; F_{\mathfrak{p}} \otimes \mathcal{O}_{X}(n P)\right)\right.
$$


L'inégalité du lemme est donc une conséquence de la définition de la stabilité.

Puisque $N(r ; d)=\max \left(N^{\prime}(r ; d) ; N_{0}\right)$ ne dépend que du genre, du rang et du degré, on termine la première partie du lemme.

Supposons maintenant que $\boldsymbol{E}$ vérifie 2) ( $\boldsymbol{E}$ sans torsion); on peut supposer que

$$
N(r ; d)>\max \left\{\mu-g ; g+r^{2}-\mu+2\right\} .
$$

Le faisceau $\boldsymbol{E}$ est localement libre : supposons qu'il existe $\mathfrak{p} \in B$ tel que $\boldsymbol{E}_{\mathfrak{p}}$ soit un faisceau avec torsion sur $X_{\mathfrak{p}}$, alors le sous-faisceau de torsion de $\boldsymbol{E}_{\mathfrak{p}}$, en ayant des sections, contredit l'hypothèse sur $\boldsymbol{E}$. On applique donc, la proposition 3.5 .

Soient $\mathfrak{p} \in B$ et $F_{\mathfrak{p}}$ l'avant-dernier fibré de la filtration de HarderNarasimhan de $\boldsymbol{E}_{\mathfrak{p}}$; le fibré $\boldsymbol{E}_{\mathfrak{p}} / F_{\mathfrak{p}}\left(\operatorname{sur} X_{\mathfrak{p}}\right.$ ) est semi-stable. Si on prouve que $\mu^{\prime}=\mu\left(F_{\mathfrak{p}}\right) \leq \mu=\mu(\boldsymbol{E})$, on aura prouvé que $\boldsymbol{E}_{\mathfrak{p}}$ est semi-stable. Soit $r^{\prime}$ le rang de $F_{\mathfrak{p}}$. On a alors ( $c f$. proposition 2.3)

$$
n+\mu+1-g \leq \frac{1}{r} h^{0}\left(X_{\mathfrak{p}} ;\left(\boldsymbol{E} \otimes \mathcal{O}_{X}(n P)\right)_{\mathfrak{p}}\right) \leq\left[\frac{\mu r-\mu^{\prime} r^{\prime}}{r-r^{\prime}}+n+1\right]_{+}
$$

d'où $\mu^{\prime} \leq \mu+(r-1) g$. Cela implique (toujours à cause de la remarque) que pour $n>g+r^{2}-\mu+2$, on a $R^{1} f_{*}\left(\boldsymbol{E} \otimes \mathcal{O}_{X}(n P)\right)=0$, d'où

$$
\begin{aligned}
\frac{1}{r} \chi\left(X_{\mathfrak{p}} ; \boldsymbol{E} \otimes \mathcal{O}_{X}(n P)\right) & =\frac{1}{r} h^{0}\left(X_{\mathfrak{p}} ;\left(\boldsymbol{E} \otimes \mathcal{O}_{X}(n P)\right)_{\mathfrak{p}}\right) \\
& \geq \frac{1}{r^{\prime}} h^{0}\left(X_{\mathfrak{p}} ;\left(F_{\mathfrak{p}} \otimes \mathcal{O}_{X}(n P)\right)\right. \\
& \geq \frac{1}{r^{\prime}} \chi\left(X_{\mathfrak{p}} ; F_{\mathfrak{p}} \otimes \mathcal{O}_{X}(n P)\right),
\end{aligned}
$$

d'où la semi-stabilité de $\boldsymbol{E}$.

Ce qui achève la démonstration du lemme 4.4.

De façon analogue on peut prouver qu'il existe un $N(r ; d) \in \mathbb{Z}$ tel que pour tout $n \geq N(r ; d)$, pour tout $\mathcal{E} \in S_{X}(r ; d)$, pour tout $\mathfrak{p} \in B$ et pour tout $F_{\mathfrak{p}}$ sous-faisceau cohérent de $\mathcal{E}_{\mathfrak{p}}$ sur $X_{\mathfrak{p}}$, les conditions suivantes sont équivalentes :

a) $\mu\left(F_{\mathfrak{p}}\right)=\mu(\mathcal{E})$;

b) $\frac{1}{r^{\prime}} h^{0}\left(X_{\mathfrak{p}} ;\left(F_{\mathfrak{p}} \otimes \mathcal{O}_{X}(n P)\right)=\frac{1}{r^{\prime}} h^{0}\left(X_{\mathfrak{p}} ;\left(\left(\mathcal{E}_{\otimes} \mathcal{O}_{X}(n P)\right)_{\mathfrak{p}}\right.\right.\right.$.

On a déjà remarqué que $S_{X}(r ; d)(\cdot) \simeq S_{X}(r ; d+r \nu)(\cdot)$ pour tout $\nu \in \mathbb{Z}$; donc on peut supposer que $d$ est assez grand pour que les propriétés suivantes soient vérifiées : pour tout $T \in \mathrm{Ob}\left(\operatorname{Sch} / B_{R}\right)$ et pour tout $\mathcal{E} \in S_{X}(r ; d)(T)$, les conditions suivantes sont satisfaites : 
1) le morphisme

$$
\operatorname{pr}_{2}^{*}\left(\operatorname{pr}_{2 ; *}(\mathcal{E})\right) \longrightarrow \mathcal{E}
$$

est surjectif $(\mathcal{E}$ est, localement sur la base, engendré par ses sections globales) et $R^{1} \operatorname{pr}_{*}(\mathcal{E})=0$ (donc $\operatorname{pr}_{2 ; *}(\mathcal{E})$ est localement libre et commute aux changements de base);

2) pour tout point fermé $t \in T(k)$ ( $k$ corps), pour tout sous-faisceau cohérent $F_{t} \subset \mathcal{E}_{t}$ (restriction de $\mathcal{E}$ à la courbe fibre $X_{t}$ ) tel que $\mu\left(F_{t}\right)=\mu(\mathcal{E})$, on a que $F_{t}$ est engendré par ses sections globales (comme faisceau sur la courbe $\left.X_{t}\right)$ et $H^{1}\left(X_{t} ; F_{t}\right)=0$.

De plus, on peut supposer que les propriétés suivantes sont vérifiés :

a) Un faisceau cohérent sur $X$ sans torsion $\boldsymbol{E} \in \operatorname{Coh}(r ; d)$ est localement libre et semi-stable si et seulement si pour tout $\mathfrak{p} \in B$ et pour tout sousfaisceau cohérent $F_{\mathfrak{p}} \subset \boldsymbol{E}_{\mathfrak{p}}$ de rang $r^{\prime}$, on a :

$$
h^{0}\left(X_{\mathfrak{p}} ; F_{\mathfrak{p}}\right) \leq \frac{r^{\prime}}{r} h^{0}\left(X_{\mathfrak{p}} ; \boldsymbol{E}_{\mathfrak{p}}\right)
$$

b) Si $\mathcal{E} \in S_{X}(r ; d)\left(B_{R}\right)$ et $\mathfrak{p} \in B$ et si $F_{\mathfrak{p}} \subset \mathcal{E}_{\mathfrak{p}}$ est de rang $r^{\prime}$, alors $F_{\mathfrak{p}}$ est de pente $\mu\left(F_{\mathfrak{p}}\right)=\mu(\mathcal{E})$ si et seulement si

$$
\frac{1}{r^{\prime}} h^{0}\left(X_{\mathfrak{p}} ; F_{\mathfrak{p}}\right)=\frac{1}{r} h^{0}\left(X_{\mathfrak{p}} ; \mathcal{E}_{\mathfrak{p}}\right) .
$$

Soient $P(n)=r n+d+r(1-g)$ le polynôme de Hilbert et $\chi=d+r(1-g)$ la caractéristique de Euler-Poincaré des éléments de $S_{X}(r ; d)$. Soit $\mathbb{Q}$ le schéma qui classifie les faisceaux quotients de $\mathcal{O}_{X}^{\chi}$ de polynôme de Hilbert $P(n)$ ( $\mathbb{Q}$ est le schéma $\left.\underline{\operatorname{Quot}}_{X / B}^{P(n)}\left(\mathcal{O}_{X}^{\chi}\right), c f .[\mathrm{G}]\right)$. On sait que $\mathbb{Q}$ est un schéma projectif sur $B_{R}$.

Le $B_{R}$-schéma en groupes réductifs $\mathrm{SL}_{\chi}=\mathrm{SL}_{\chi}\left(\mathcal{O}_{B_{R}}\right)$ agit linéairement sur le schéma $\mathbb{Q}$. On peut décrire cette action de la façon suivante : si $\mathcal{F}=\mathcal{O}_{X}^{\chi} / \mathcal{W}$ est un quotient de $\mathcal{O}_{X}^{\chi}$ «représenté par $\mathbb{Q}$ » et $g \in S L_{\chi}$, alors le transformé de $\mathcal{F}$ par $g$ est $\mathcal{O}_{X}^{\chi} / g^{-1} \mathcal{W}$.

Sur $X \times{ }_{B} \mathbb{Q}$ il existe un quotient universel $\mathcal{U}$ du faisceau $\mathcal{O}_{X \times_{B_{R}} \mathbb{Q}}^{\chi}$.

Pour tout $B$-schéma $T$ et pour tout $q \in \mathbb{Q}(T)$, on indiquera par $F_{q}$ le faisceau $g^{*}(\mathcal{U})$ sur $X_{T}$ où $g$ est donnée par le diagramme cartésien suivant

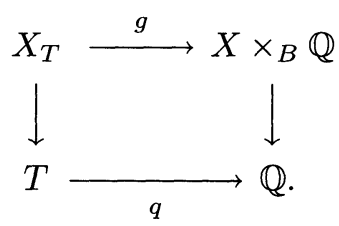

$\left(\right.$ On a $X_{T}=X \times_{B} T$.) 
Soient $t \in B_{R}$ un point géométrique de $B_{R}$ et $k(t)$ le corps de définition de $t$ (on supposera toujours que $k(t)$ est parfait).

Si $A$ est un $R$-module libre, on note $\mathrm{Gr}^{m}(A)$ la grassmannienne des $R$-modules libres de rang $m$ quotients de $A\left(\mathrm{Gr}^{m}(A)\right.$ est un $R$-schéma projectif et lisse).

Pour tout $n$ assez grand, on a un plongement $\mathrm{SL}_{\chi}$-équivariant

$$
\mathbb{Q} \longrightarrow \operatorname{Gr}^{r n+\chi}\left(f_{*}\left(\mathcal{O}_{X}(n P)\right)^{\oplus \chi}\right)
$$

qui à $\mathcal{E}$ associe le quotient $f_{*}\left(\mathcal{E} \otimes \mathcal{O}_{X}(n P)\right)$. Ce qui permet d'étudier les points semi-stables de $\mathbb{Q}$ sous l'action de $\mathrm{SL}_{\chi}$.

Un théorème à la base de la construction de l'espace des modules des fibrés semi-stables sur une courbe ( $c f$. par exemple [LP, p. 109]) affirme que :

Si $r$ et $d$ vérifient les propriétés 1), 2), a) et b), alors pour tout $q \in \mathbb{Q}(\mathcal{L})\left(k_{\mathfrak{p}}\right)$, on a l'équivalence :

1) le faisceau $F_{q}$ sur $X_{t}$ est semi-stable et l'application naturelle

$$
\left(\mathcal{O}_{K}^{\chi}\right)_{t} \longrightarrow H^{0}\left(X_{t} ; F_{q}\right)
$$

induite par la projection canonique, est un isomorphisme;

2) le point $q$ est semi-stable sous l'action de $\left(\mathrm{SL}_{\chi}\right)_{t}$.

Soit $\mathbb{Q}^{s s}$ l'ouvert des points semi-stables sous l'action du schéma en groupes $\mathrm{SL}_{\chi}$. Alors le $B_{R}$-schéma qu'on cherche est :

$$
\mathfrak{U}_{X}(r ; d)=\mathbb{Q}^{s s} / \mathrm{SL}_{\chi}
$$

On remarque que, les observations après la proposition 4.2 impliquent que, par construction, pour tout $\mathfrak{p} \in B_{R}$ (p est un idéal maximal de $R$ où le «corps des fractions de $R$ ») le $k(\mathfrak{p})$-schéma $\mathfrak{U}_{X}(r ; d)_{\mathfrak{p}}$ (fibre au-dessus de $\mathfrak{p})$ est l'espace de modules des fibrés semi-stables de rang $r$ et degré $d$ sur la $k(\mathfrak{p})$-courbe $X_{\mathfrak{p}}$.

Prouvons maintenant la propriété d'espace de modules grossier de $\mathfrak{U}_{X}(r ; d)$. Remarquons d'abord qu'une conséquence du critère valuatif de séparation est que, si $X$ et $Y$ sont deux $B_{R}$-schémas avec $Y$ séparé sur $B_{R}$, $X_{K}$ schématiquement dense dans $X$, et $f$ et $g$ sont deux $B_{R^{-m o r p h i s m e s}}$ de $X$ vers $Y$, alors $f=g$ si (et seulement si) $f_{K}=g_{K}, f_{K}$ (resp. $g_{K}$ ) étant la restriction de $f$ (resp. $g$ ) à la fibre générique.

Soit $T$ un $B_{R}$-schéma séparé et $\mathcal{E}$ un fibré sur $X_{T}=X \times_{B_{R}} T$ semistable sur chaque fibre géometrique de la projection $\operatorname{pr}_{2}: X_{T} \rightarrow T$. On

TOME $125-1997-\mathrm{N}^{\circ} 4$ 
suppose que le degré (sur chaque fibre) de $\mathcal{E}$ est assez grand. Le fibré $\operatorname{pr}_{2, *}(\mathcal{E})$ sur $T$ est donc localement libre.

Soit $\mathcal{U}=\left\{U_{\alpha}\right\}_{\alpha \in A}$ un recouvrement par ouverts de $T$ tel que, pour tout $\alpha \in A, \operatorname{pr}_{2, *}(\mathcal{E})_{\mid U_{\alpha}}$ est libre sur $U_{\alpha}$.

Sur $X \times{ }_{B_{R}} U_{\alpha}$ on a un morphisme surjectif

$$
\mathcal{O}_{X \times_{B_{R}} U_{\alpha}}^{\chi} \longrightarrow \mathcal{E}_{\mid X \times_{B_{R}} U_{\alpha}}
$$

donc, par fonctorialité, on trouve un $B_{R}$-morphisme

$$
\varphi_{\alpha}: U_{\alpha} \longrightarrow \mathbb{Q}
$$

L'étude sur la stabilité que l'on a faite implique alors que l'image de $\varphi_{\alpha}$ est contenue dans l'ouvert $\mathbb{Q}^{s s}$ de $\mathbb{Q}$. On trouve donc un morphisme

$$
\psi_{\alpha}: U_{\alpha} \longrightarrow \mathfrak{U}_{X}(r ; d)
$$

Pour $\alpha$ et $\beta \in A$, les morphismes $\psi_{\alpha}$ et $\psi_{\beta}$ coïncident sur $U_{\alpha} \cap U_{\beta}$ car leur restriction à la fibre générique coïncident (propriété d'espace de modules grossier de $\mathfrak{U}_{X_{K}}(r ; d)$ ). Les $\psi_{\alpha}$ définissent alors (par recollement de morphismes) un morphisme

$$
\psi: T \rightarrow \mathfrak{U}_{X}(r ; d)
$$

De plus, on observe que, pour chaque $\mathfrak{p} \in B_{R}$, le morphisme $\psi_{\mathfrak{p}}: T_{\mathfrak{p}} \rightarrow$ $\mathfrak{U}_{X}(r ; d)_{\mathfrak{p}}$ est le morphisme obtenu par la famille $\mathcal{E}_{\mathfrak{p}}$ sur le $k(\mathfrak{p})$-schéma $\left(X \times_{B_{R}} T\right)_{\mathfrak{p}}$.

La deuxième propriété pour avoir un espace de modules grossier est évidente : il suffit de prendre $T=\mathbb{Q}^{s s}$ et d'appliquer la propriété de quotient catégorique de $\mathfrak{U}_{X}(r ; d)$.

Pour ce qui concerne la bijection entre $\operatorname{St}_{X}(r ; d)(k)$ et $U_{s t}(k)$ il suffit d'appliquer la propriété 5 de la proposition 4.2 .

Ce qui achève la démonstration du théorème 4.3.

Proposition 4.5. - Le schéma $\mathfrak{U}_{X}(r ; d)$ est plat sur $B_{R}$.

Preuve. - On a déjà remarqué ( $c f$. remarque après la prop. 4.2) que la construction du quotient commute aux changements de base plats; on remarque aussi que la Proposition 4.2 reste valable même si $R$ est le completé d'un anneau de valuation discréte ( $c f$. [S]); donc, puisque la platitude est une propriété locale sur la base, on peut supposer que $R$ est local. On complète $R$. 
On suppose donc que $B=\operatorname{Spec}(R)$, où $R$ est un anneau de valuation discrète complet.

On peut supposer $d$ assez grand.

Il suffit de prouver que $\mathfrak{U}_{X}(r ; d)$ est irréductible. En effet, la fibre spéciale de $\mathfrak{U}_{X}(r ; d)$ est intègre ( $c f$. [SD, chap. 1]), donc réduite (la fibre générique aussi); par conséquent, si $\mathfrak{U}_{X}(r ; d)$ est irréductible, le faisceau d'idéaux des éléments nilpotents est nul par le lemme de Nakayama; donc $\mathfrak{U}_{X}(r ; d)$ est intègre, d'où la platitude.

Soit $J_{X / B}^{d}$ la composante du schéma de Picard $\underline{\mathrm{Pic}}_{X / B}$ paramétrisant les faisceaux inversibles sur $X$ de degré $d$ sur les fibres. Soit $\mathcal{P}$ un faisceau de Poincaré sur $X \times_{B} J_{X / B}^{d}$. Si $\mathrm{pr}_{2}: X \times_{B} J_{X / B}^{d} \rightarrow J_{X / B}^{d}$ est la projection naturelle, le faisceau $R^{1} \operatorname{pr}_{2 *}\left(\mathcal{P}^{-1}\right)$ est localement libre.

Soient

$$
\mathcal{F}=R^{1} \operatorname{pr}_{2 *}\left(\mathcal{P}^{-1}\right) \otimes \mathcal{O}_{J_{X / B}^{d}}^{r-1} \quad \text { et } \quad \mathcal{H}=\operatorname{Spec}(\operatorname{Sym}(\mathcal{F}))
$$

$\mathcal{H}$ classifie les extensions de $\mathcal{P}$ par $\mathcal{O}_{X \times{ }_{B} J_{X / B}^{d}}^{r-1}$.

Soit $\mathbb{E}$ l'extension universelle de $\mathcal{P}$ par $\mathcal{O}_{X \times{ }_{B} J_{X / B}^{d}}^{r-1}$ sur $X \times_{B} \mathcal{H}$ (une telle extension existe car $\operatorname{pr}_{2, *}\left(\mathcal{P}^{-1}\right)=0$, cf. [NR] ou [R]), et soit $\mathcal{U}$ l'ouvert de $\mathcal{H}$ où $\mathbb{E}_{\mid \mathcal{U}}$ est semi-stable sur chaque fibre de la projection $p: X \times_{B} \mathcal{H} \rightarrow \mathcal{H}$. La famille $\mathbb{E}_{\mid \mathcal{U}}$ définit donc (par fonctorialité) un morphisme

$$
\mathcal{U} \longrightarrow \mathfrak{U}_{X}(r ; d) .
$$

Ce morphisme est surjectif sur les points fermés de $\mathfrak{U}_{X}(r ; d)$ grâce au lemme suivant.

Lemme 4.6. - Soit $X$ une courbe sur un corps $k$ et $F$ un fibré vectoriel engendré par ses sections globales, alors il existe une suite exacte

$$
0 \rightarrow \mathcal{O}_{X}^{r g(F)-1} \longrightarrow F \longrightarrow \operatorname{det}(F) \rightarrow 0 .
$$

Pour une démonstration regarder, par exemple [SD, lemme 24, p. 27].

Mais $\mathcal{U}$ est irréductible (car $J_{X / B}$ et $\mathcal{H}$ le sont), donc $\mathfrak{U}_{X}(r ; d)$ aussi.

On peut maintenant généraliser cette construction au cas où $R=\mathcal{O}_{K}$, ou, plus généralement, au cas où $\operatorname{Pic}(R)$ n'est pas trivial.

ThÉORÈme 4.7. - Soient $r$ et $d$ deux entiers (avec $r>0$ ); alors il existe un schéma $g: \mathfrak{U}_{X}(r ; d) \rightarrow B$, qui est un espace de modules grossier pour le foncteur $S_{X}(r ; d)$. Le morphisme $g$ est projectif et plat.

Preuve. - La principale difficulté ici, est que, si $\mathcal{E}$ et $\mathcal{E}^{\prime}$ sont deux fibrés sur $X$, semi-stables sur chaque fibre et de même rang et même degré, on

TOME $125-1997-\mathrm{N}^{\circ} 4$ 
ne peut pas dire qu'il existe un isomorphisme $f_{*}(\mathcal{E}) \rightarrow f_{*}\left(\mathcal{E}^{\prime}\right)$, même si leur degré est très grand. En effet, deux modules projectifs de même rang sur un anneau avec groupe de Picard non trivial, ne sont pas, a priori, isomorphes.

On peut résoudre le problème de la façon suivante : on suppose $d$ assez grand pour que les propriétés 1 ), 2), a) et b) du théorème 4.3 soient vérifiées.

Soit $\boldsymbol{Q}$ le $B$-schéma $\underline{\operatorname{uot}}_{X / B}^{P(n)}\left(\mathcal{O}_{X}^{\chi}\right)$. Sur $\boldsymbol{Q}$, on a l'action linéaire du schéma en groupes réductifs sur $B, \mathrm{SL}_{\chi}=\mathrm{SL}_{\chi}\left(\mathcal{O}_{K}\right)$ (on linéarise l'action de $\mathrm{SL}_{\chi}$ sur $\boldsymbol{Q}$ comme dans la preuve du théorème 4.3 ).

On pose alors

$$
\mathfrak{U}_{X}(r ; d)=\boldsymbol{Q} / S L_{\chi}
$$

Soit $\mathcal{V}=\left\{V_{\alpha}\right\}_{\alpha \in I}$ un recouvrement de $B$ par ouverts affines tels que $V_{\alpha}=\operatorname{Spec}\left(R_{\alpha}\right)$ avec $\operatorname{Pic}\left(R_{\alpha}\right)$ trivial pour tout $\alpha \in I$.

On sait que $\mathfrak{U}_{X}(r ; d)$ est un quotient catégorique uniforme de $\boldsymbol{Q}$ (cf. la remarque après la proposition 4.1 ), donc il commute aux changements de base plats. Cela implique que

$$
\mathfrak{U}_{X_{U_{\alpha}}}(r ; d)=\mathfrak{U}_{X}(r ; d)_{\mid U_{\alpha}} .
$$

Soit $\mathcal{E}$ une famille de fibrés semi-stables sur $X$ paramétrisée par un schéma $T$ (séparé sur $B$ ); soit $T_{\alpha}$ la restriction de $T$ à $U_{\alpha}$; il existe donc un morphisme

$$
\psi_{\alpha}: T_{\alpha} \longrightarrow \mathfrak{U}_{X}(r ; d)_{\left.\right|_{\alpha}}
$$

Pour tout $\alpha$ et $\beta \in I$, les morphismes $\psi_{\alpha}$ et $\psi_{\beta}$ coïncident sur $T_{\alpha} \cap T_{\beta}$ (car ils coïncident sur la fibre générique et sur chaque fibre spéciale); donc les $\psi_{\alpha}$ se recollent; on a donc un morphisme

$$
\psi_{\mathcal{E}}: T \longrightarrow \mathfrak{U}_{X}(r ; d)
$$

On vérifie de façon analogue les autres propriétés d'espace de modules grossier. Ce qui termine la démonstration.

REMARQUe. - D'après la démonstration, on voit que :

a) $\mathfrak{U}_{X}(r ; d)_{K} \simeq \mathfrak{U}_{X_{K}}(r ; d)$; il en résulte que $\mathfrak{U}_{X}(r ; d)$ est un modèle canonique de l'espace de modules des fibrés semi-stable de rang $r$ et de degré $d$ sur la courbe algébrique $X_{K}$.

b) Le morphisme $g$ est de dimension relative $r^{2}\left(g\left(X_{K}\right)-1\right)+1$.

Remarque. - Dans la suite, si $T \in \mathrm{Ob}(\mathrm{Sch} / B)$ et $\mathcal{E} \in S_{X}(r ; d)(T)$, on notera $\psi_{\mathcal{E}}: T \rightarrow \mathfrak{U}_{X}(r ; d)$ le $B$-morphisme définit par $\mathcal{E}$. 


\section{La famille universelle}

On supposera à partir de maintenant dans cet article que $r$ et $d$ sont deux entiers fixés, premiers entre eux. On suppose $d$ assez grand fixé comme dans le théorème 4.3. On se propose, maintenant, de construire un fibré $\mathcal{E}$ sur $X \times_{B} \mathfrak{U}_{X}(r ; d)$ de rang $r$, tel que pour tout $T \in \mathrm{Ob}(\operatorname{Sch} / B)$ et pour tout $P \in \mathfrak{U}_{X}(r ; d)(T)$, le fibré $\mathcal{E}_{P}=(\text { id } \times P)^{*} \mathcal{E}$ soit dans $S_{X}(r ; d)$ et $\psi_{\mathcal{E}_{P}}=P$.

Puisque $r$ et $d$ sont premiers entre eux et $\mathfrak{U}_{X}(r ; d)$ est plat $\operatorname{sur} B$, on a que $\mathfrak{U}_{X}(r ; d)$ est lisse sur $B$. En effet, pour chaque $\mathfrak{p} \in B,\left(\mathfrak{U}_{X}(r ; d)\right)_{\mathfrak{p}}$ est isomorphe à $\mathfrak{U}_{X_{\mathfrak{p}}}(r ; d)$ qui est lisse sur $k_{\mathfrak{p}}$ (cf. par exemple [SD, chap. 1]).

On observe que, puisque $r$ et $d$ sont premiers entre eux, tout fibré semistable de rang $r$ et degré $d$ est stable : en effet, il suffit de remarquer que, si $X$ est une courbe lisse sur un corps et $\mathcal{E}$ est un fibré semi-stable de rang $r$ et degré $d$ sur $X$, alors $\mathcal{E}$ est stable sur $X$. Soit $F$ un sous-fibré de $\mathcal{E}$ de rang $r^{\prime}<r$ et de degré $d^{\prime}$ : alors $d^{\prime} r<d r^{\prime}$; en effet, si $d^{\prime} r=d r^{\prime}$, alors $d^{\prime} r=d r^{\prime}<d r$, donc $d^{\prime}<d$; mais $d \mid d^{\prime} r$ et donc $d \mid d^{\prime}$ et c'est absurde.

Remarque. - On rappelle qu'on a fixé une section $P$ de $f$ donc $S_{X}(1 ; d)(\cdot)$ coïncide avec le foncteur $\operatorname{Pic}_{X / B}^{d}(c f .[\mathrm{BLR}])$. On vérifie aisément, à l'aide des propositions 3.3 et 3.5, que, si $r$ et $d$ sont premiers entre eux, $S_{X}(r ; d)(\cdot)$ est un faisceau pour la topologie de Zariski.

Proposition 5.1. - Soient $r$ et d deux entiers premiers entre eux. Il existe un fibré $\mathcal{E}$ de rang $r$ sur $\mathfrak{U}_{X}(r ; d) \times_{B} X$ tel que, pour tout $B$ schéma $T$ et pour tout $P \in \mathfrak{U}_{X}(r ; d)(T)$, le fibré sur $X \times_{B} T$ obtenu par le diagramme cartésien

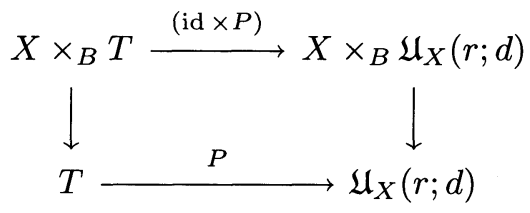

est dans $S_{X}(r ; d)(T)$.

De plus, si $\boldsymbol{E}$ est un élément de $S_{X}(r ; d)(T)$, alors il existe $\mathcal{N} \in \operatorname{Pic}(T)$ tel que

$$
\left(\operatorname{id} \times \psi_{\boldsymbol{E}}\right)^{*}(\mathcal{E}) \simeq \boldsymbol{E} \otimes p_{2}^{*}(\mathcal{N})
$$

$\left(p_{2}: X_{T} \rightarrow T\right.$ étant la deuxième projection). Ou, de façon plus syntétique, le couple $\left(\mathfrak{U}_{X}(r ; d) ; \mathcal{E}\right)$ représente le foncteur $S_{X}(r ; d)$.

Preuve. - Soit $\boldsymbol{Q}$ le schéma Quot introduit dans la démonstration du théorème 4.7 et soit $\Omega=Q^{s s} \subset \bar{Q}$ l'ouvert des points stables (ou semistables qui est le même) et $\mathcal{U}$ un fibré universel sur $X \times_{B} \Omega$.

$$
\text { TOME } 125-1997-\mathrm{N}^{\circ} 4
$$


On remarque que l'action de $\mathrm{SL}_{\chi}$ sur $\boldsymbol{Q}$ provient d'une action de $\mathrm{GL}_{\chi}\left(\mathcal{O}_{K}\right)$.

Soit $\mathbb{T}$ le centre de $\mathrm{GL}_{\chi}\left(\mathcal{O}_{K}\right)$; on voit aisément qu'il agit trivialement sur $\Omega$; on en déduit une action du $B$-schéma en groupes $G=\mathrm{GL}_{\chi}\left(\mathcal{O}_{K}\right)$ $\operatorname{sur} \Omega$.

Un lemme classique nous assure que pour tout $p \in \Omega(k)$ (où $k$ est un corps avec un morphisme $\mathcal{O}_{K} \rightarrow k$ ) le stabilisateur de $p$ dans $G$ est le groupe des automorphismes de $\mathcal{U}_{\mid X \times_{k}\{p\}}$ (cf. [LP, lemme 8.13, p. 128]).

L'action de $G$ sur $\Omega$ se factorise alors par une action de $\operatorname{PGL}\left(\mathcal{O}_{B}^{\chi}\right)=$ PGL.

L'action de $G$ sur $\mathcal{U}$ n'induit pas une action de PGL. En effet, le centre de $G, \mathbb{T} \simeq \mathbb{G}_{m}$ n'agit pas trivialement sur $\mathcal{U}$ : il agit par homothéties.

Pour avoir une action de PGL sur un fibré universel sur $X \times \Omega$, on tensorise le fibré $\mathcal{U}$ par un faisceau $p_{2, *}\left(\mathcal{M}^{-1}\right)$, où $\mathcal{M}$ est un fibré inversible sur $\Omega$ sur lequel $G$ agit linéairement et l'action du centre $\mathbb{T}$ étant celle de $\mathbb{G}_{m}$ par homothétie.

En effet, si $G$ agit sur $\mathcal{M}$ de façon telle que l'action de $\mathbb{T} \simeq \mathbb{G}_{m}$ sur $\mathcal{M}$ soit celle de $\mathbb{G}_{m}$ par homothétie, alors PGL agit sur $\mathcal{U} \otimes p_{2}^{*}\left(\mathcal{M}^{-1}\right)$ de façon évidente.

Soit $\mathcal{O}_{X}(P)$ le fibré inversible associé à la section fixée au début du troisième paragraphe. Les faisceaux

$$
\mathcal{F}_{1}=p_{2 *}(\mathcal{U}) \quad \text { et } \quad \mathcal{F}_{2}=p_{2 *}\left(\mathcal{U} \otimes p_{1}^{*}\left(\mathcal{O}_{X}(P)\right)\right)
$$

sont localement libres de rangs respectivement $a=d+r(1-g)$ et $b=d+r(2-g)$. Le $\mathcal{O}_{K^{-}}$-schéma en groupes $G$ agit sur chacun d'eux et l'action de $\mathbb{T}$ est celle de $\mathbb{G}_{m}$ par homothétie. Soient $u$ et $v$ tels que $a u+b v=1$; alors on pose :

$$
\mathcal{M}=\left(\operatorname{det}\left(\mathcal{F}_{1}\right)\right)^{\otimes u} \otimes\left(\operatorname{det}\left(\mathcal{F}_{2}\right)\right)^{\otimes v} .
$$

On pose $\mathbb{F}=\mathcal{U} \otimes p_{2}^{*}\left(\mathcal{M}^{-1}\right)$. Sur $\mathbb{F}$ on a une action linéaire de $G$, l'action de $\mathbb{T}$ étant triviale.

On a, donc, construit un fibré $\mathbb{F}$ sur $X \times_{B} \Omega$ et une action de PGL sur $\mathbb{F}$ compatible à l'action de PGL sur $X \times_{B} \Omega$ : cela signifie qu'on a un diagramme commutatif

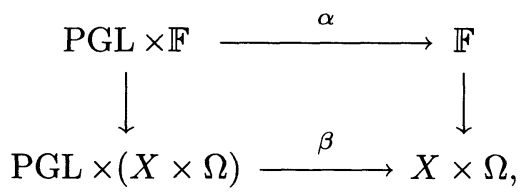

$\alpha$ étant l'action de PGL sur $\mathbb{F}$ et $\beta$ celle sur $X \times \Omega$.

BULLETIN DE LA SOCIÉTÉ MATHÉMATIQUE DE FRANCE 
Remarque. - Il est plus facile, dans ce cadre, de regarder $\mathbb{F}$ comme un fibré sur $X \times \Omega$ (à savoir un schéma sur $X \times \Omega$ qui est localement $X \times \Omega \times \mathbb{A}^{r}$, etc.) que comme un faisceau localement libre (les deux façons étant équivalentes).

Le morphisme $\vartheta: X \times_{B} \Omega \rightarrow X \times_{B} \mathfrak{U}_{X}(r ; d)$ est plat : en effet $X \times_{B} \mathfrak{U}_{X}(r ; d)$ est plat sur $B$ et pour tout $\mathfrak{p} \in B$ le morphisme $\vartheta_{\mid \mathfrak{p}}$ est plat, donc $\vartheta$ est plat pour SGA 1.IV.5.9. Le morphisme

$$
\gamma: \Omega \times \mathrm{PGL} \longrightarrow \Omega \times_{\mathfrak{U}_{X}(r ; d)} \Omega
$$

est un isomorphisme : $\Omega \times_{\mathfrak{U}_{X}(r ; d)} \Omega$ est plat sur $B$ et pour tout $\mathfrak{p} \in B$, $\gamma_{\mid \mathfrak{p}}$ est un isomorphisme ( $c f$. [SD, chap. 1, p. 37]) donc $\gamma$ est un isomorphisme pour SGA 4, cor. 17.9.5.

Cela entraîne qu'on peut appliquer la théorie de la descente pour morphismes fidèlement plats (voir $[G]$ ) et en déduire l'existence d'un fibré $\mathcal{E}$ sur $X \times_{B} \mathfrak{U}_{X}(r ; d)$ tel que $\vartheta^{*}(\mathcal{E}) \simeq \mathcal{U} \otimes p_{2}^{*}(\mathcal{M})$. En effet, par exemple, du diagramme cartésien

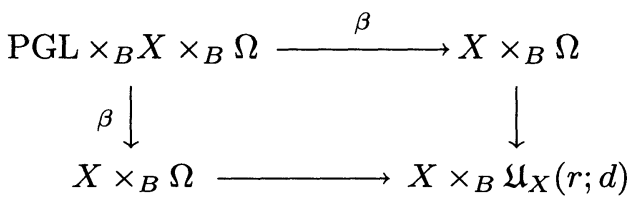

on en déduit un isomorphisme $p^{*} \mathbb{F} \simeq \beta^{*} \mathbb{F}$ (les autres isomorphismes pour avoir une donnée de descente sont aussi élémentaires à construire).

$\grave{A}$ partir de la construction, on peut observer que pour tout $\mathfrak{p} \in B$, le fibré $\mathcal{E}_{\mid \mathfrak{p}}$ est la famille universelle sur $X_{\mathfrak{p}} \times \mathfrak{U}_{X_{\mathfrak{p}}}(r ; d)$

La deuxième partie est évidente grâce à la proposition 3.5 .

Métriques sur la famille universelle

Soient maintenant $X$ une surface de Riemann de genre $g \geq 1$ et $\mathrm{d} \mu_{\mathrm{Ar}}$ la métrique d'Arakelov sur $X$. La métrique $\mathrm{d} \mu_{\mathrm{Ar}}$ est donnée par :

$$
\mathrm{d} \mu_{\mathrm{Ar}}=\frac{i}{2 g} \sum_{i=1}^{g} \omega_{i} \wedge \bar{\omega}_{i}
$$

où $\omega_{1}, \ldots, \omega_{g}$ est une base de $H^{0}\left(X ; \Omega_{X}^{1}\right)$ orthonormée par rapport au produit hermitien

$$
(\alpha ; \beta)=\frac{i}{2} \int_{X} \alpha \wedge \bar{\beta} .
$$

TOME $125-1997-\mathrm{N}^{\circ} 4$ 
Soient $r$ et $d$ deux entiers premiers entre eux, $\mathfrak{U}_{X}(r ; d)$ l'espace des modules des fibrés stables de rang $r$ et degré $d$ sur $X$, et soit $\mathbb{E}$ une famille universelle sur $X \times \mathfrak{U}_{X}(r ; d)$.

Soit $E$ un fibré holomorphe sur $X$; on rappelle qu'une métrique $h$ sur $E$ est dite d'Einstein-Hermite par rapport à la métrique d'Arakelov si

$$
\Lambda F=\lambda \text { id pour } \lambda \in \mathbb{C}
$$

lorsqu'on dénote par $F$ la courbure de $h$ et par $\Lambda$ la adjointe de l'accouplement «cup» par $\mathrm{d} \mu_{\mathrm{Ar}}$ sur les formes à coefficients dans $\operatorname{End}(E)$.

Plus explicitement et plus généralement $(c f .[\mathrm{Ko}]):$ si $s=\left(s_{1} ; \ldots ; s_{r}\right)$ sont des coordonnées locales pour $E$ et $z$ est une coordonnée locale sur $X$, alors la courbure de $h$ est donnée par

$$
R\left(s_{j}\right)=\sum_{i=1}^{r} \Omega_{j}^{i} s_{i}
$$

où les $\Omega_{j}^{i}$ sont des $1-1$ formes sur $X ;$ donc

$$
\Omega_{j}^{i}=R_{j}^{i}(z) \mathrm{d} z \wedge \mathrm{d} \bar{z}
$$

avec $R_{j}^{i}(z)$ fonction $C^{\infty}$. Soit $g=g_{1,1}(z) \mathrm{d} z \mathrm{~d} \bar{z}$ une métrique sur $X$. La condition d'être une métrique d'Einstein-Hermite par rapport à $g$ est équivalent à :

$$
R_{j}^{i}(z)=\lambda g_{1 ; 1}(z) \delta_{j}^{i}
$$

pour une constante $\lambda$ (ici, $\delta_{j}^{i}$ est le symbole de Kronecker).

On dira que $(E ; h)$ est un fibré métrisé d'Einstein-Hermite par rapport à la métrique $g$ sur $X$.

Quelques remarques sur la notion de métrique d'Einstein-Hermite sont nécessaires :

a) Deux métriques d'Einstein-Hermite sont proportionnelles.

b) Si $E$ est un fibré inversible sur $X$, alors une métrique sur $E$ est d'Einstein-Hermite si et seulement si elle est permise ( $c f$. [MB] ou [Ga]).

c) (Donaldson) Un fibré holomorphe $\mathcal{E}$ sur $X$ admet une métrique d'Einstein-Hermite si et seulement si il est poly-stable (somme directe de fibrés stables de même pente ( $c f$. [Do])).

Donc on voit que, la notion de métrique d'Einstein-Hermite, d'une part généralise au rang supérieur la notion de métrique permise, et d'autre part elle est l'analogue analytique de la notion de stabilité pour les fibrés en géométrie algébrique. 
Proposition 5.2. - Soient $r \geq 1$ et d deux entiers premiers entre eux. Soit $\mathbb{E}$ une famille universelle sur $X \times \mathfrak{U}_{X}(r ; d)$. Il existe une métrique $h$, $C^{\infty}$ sur $\mathbb{E}$, telle que, pour tout $q \in \mathfrak{U}_{X}(r ; d)$ la métrique sur le faisceau, stable sur $X, \mathbb{E}(q)=\mathbb{E}_{\mid X \times\{q\}}$ obtenue par restriction de la métrique $h$, soit d'Einstein-Hermite par rapport à $\mathrm{d} \mu_{\mathrm{Ar}}$.

Preuve. - On remarque d'abord qu'on peut réduire le problème à un problème local sur $\mathfrak{U}_{X}(r ; d)$ : en effet, si $h$ est une métrique sur $\mathbb{E}$ qui est d'Einstein-Hermite sur chaque fibre et $g$ est une fonction $C^{\infty}$ sur $\mathfrak{U}_{X}(r ; d)$, alors $g h$ est aussi une métrique qui est d'Einstein-Hermite sur chaque fibre. Supposons qu'on a trouvé un recouvrement $\mathcal{U}=\left\{U_{i}\right\}_{i \in I}$ de $\mathfrak{U}_{X}(r ; d$ ) (fini car $\mathfrak{U}_{X}(r ; d)$ est projectif donc compact) et $h_{i}$ des métriques sur $\mathbb{E}_{\mid X \times U_{i}}$ d'Einstein-Hermite sur chaque fibre; soit $\left\{g_{i}\right\}$ une partition de l'unité sur $\mathfrak{U}_{X}(r ; d)$ par rapport à $\mathcal{U}$, alors la métrique $\sum_{i} g_{i} h_{i}$ est une métrique sur $\mathbb{E}$ qui vérifie la propriété qu'on cherche (deux métriques d'Einstein-Hermite sont proportionnelles, donc $h_{i} / h_{j}$ est une fonction $C^{\infty}$ sur $U_{i} \cap U_{j}$ ).

Donc, soit $\mathbb{D}$ un disque dans $\mathbb{C}^{n}$ et $\boldsymbol{E}$ un fibré holomorphe de rang $r$ sur $X \times \mathbb{D}$ stable sur chaque fibre de la projection $p: X \times \mathbb{D} \rightarrow \mathbb{D}$. En utilisant le théorème de Donaldson (chaque fibré stable sur $X$ admet une métrique d'Einstein-Hermite $c f$. [Do]) et le théorème (4.21), p. 266 dans [Ko] (l'espace de modules des connections d'Einstein-Hermite sur $(\boldsymbol{E} ; h)$ est ouvert dans l'espace de modules des structures holomorphes sur $\boldsymbol{E}$ ) on peut trouver la métrique qu'on cherche sur $\boldsymbol{E}$.

\section{Hauteurs sur $\mathfrak{U}_{\boldsymbol{X}}(\boldsymbol{r} ; \mathcal{F})$ et sur $\mathfrak{U}_{\boldsymbol{X}_{\boldsymbol{K}}}\left(\boldsymbol{r} ; \mathcal{F}_{\boldsymbol{K}}\right)$}

Supposons que $r>1$ et $d$ soient deux entiers premiers entre eux.

On se propose d'étudier l'arithmétique des fibrés stables de rang $r$ et degré $d$ sur $X$. L'espace $\mathfrak{U}_{X}(r ; d)$ semble, pour l'instant être trop grand; on restreint donc notre attention aux fibrés de rang et de déterminant fixés.

Soit $\mathcal{F}$ un fibré inversible sur $X$ de degré $d$ sur chaque fibre de $f$ et soit $\mathcal{F}_{K}$ la restriction de $\mathcal{F}$ à la fibre générique $X_{K}$.

Soit $T$ un $B$-schéma et $\mathcal{E}$ un fibré sur $X_{T}=X \times_{B} T$, on dira que $\mathcal{E}$ possède la propriété $\left.{ }^{* *}\right)$ si la condition suivante est vérifiée :

$$
\left\{\begin{array}{l}
\text { Il existe un recouvrement ouvert } \mathcal{U}=\left\{U_{i}\right\}_{i \in I} \text { de } T \text { tel que } \\
\text { pour tout } i \in I \text { on } \operatorname{det}\left(\mathcal{E}_{i}\right)=\operatorname{det}\left(\mathcal{E}_{\mid X \times U_{i}}\right) \simeq \mathcal{F}_{i}=\mathcal{F}_{\mid X \times U_{i}}
\end{array}\right.
$$

On considère le foncteur

$$
S_{X}(r ; \mathcal{F})(\cdot): \operatorname{Sch} /{ }_{B} \longrightarrow \mathcal{E} n s
$$

TOME $125-1997-\mathrm{N}^{\circ} 4$ 
qui à chaque $T \in \mathrm{Ob}(\mathrm{Sch} / B)$ associe l'ensemble

$$
\begin{aligned}
S_{X}(r ; \mathcal{F})(T)=\left\{\mathcal{E} \mid \mathcal{E} \text { est un fibré sur } X \times_{B_{R}} T=X_{T}\right. \\
\text { qui vérifie } \left.\left({ }^{*}\right) \text { et }\left({ }^{* *}\right)\right\} / \sim
\end{aligned}
$$

où la condition $\left(^{*}\right)$ et la relation d'équivalence $\sim$ ont été déjà definies dans le paragraphe 3 .

Si $h: T^{\prime} \rightarrow T$ est un $B$-morphisme, alors on a une application

$$
(\mathrm{id} \times h)^{*}: S_{X}(r ; \mathcal{F})(T) \rightarrow S_{X}(r ; \mathcal{F})\left(T^{\prime}\right)
$$

donc $S_{X}(r ; \mathcal{F})(\cdot)$ est bien un foncteur contravariant.

On obtient la représentabilité de ce foncteur comme corollaire du théorème 4.7 et de la proposition 5.1 :

ThÉORÈme 6.1. - Il existe un schéma $g_{\mathcal{F}}: \mathfrak{U}_{X}(r ; \mathcal{F}) \rightarrow B$ qui représente le foncteur $S_{X}(r ; \mathcal{F})$ et tel que :

a) $\mathfrak{U}_{X}(r ; \mathcal{F})_{K} \simeq \mathfrak{U}_{X_{K}}\left(r ; \mathcal{F}_{K}\right)$;

b) Le morphisme $g_{\mathcal{F}}$ est projectif, lisse et de dimension relative égale $\grave{a}$ $\left(r^{2}-1\right)\left(g\left(X_{K}\right)-1\right)$.

Remarque. - L'espace $\mathfrak{U}_{X}(r ; \mathcal{F})$ est donc un modèle «canonique» de l'espace de modules des fibrés semi-stable de rang $r$ et déterminant isomorphe à $\mathcal{F}_{K}$ sur la courbe algébrique $X_{K}$.

Preuve. - Il est évident que $S_{X}(r ; \mathcal{F})$ est un sous-foncteur de $S_{X}(r ; d)$.

Soit $J_{X / B}^{d}$ la composante du schéma de Picard $\underline{\mathrm{Pic}}_{X / B}$ paramétrisant les faisceaux inversibles sur $X$ de degré $d$ sur chaque fibre. Soit $\mathbb{E}$ la famille universelle sur $X \times \mathfrak{U}_{X}(r ; d)$.

Le faisceau inversible $\operatorname{det}(\mathbb{E})$ sur $X \times \mathfrak{U}_{X}(r ; d)$ permet de construire un morphisme $\operatorname{det}: \mathfrak{U}_{X}(r ; d) \rightarrow J_{X / B}^{d}$.

Le fibré inversible $\mathcal{F}$ détermine un point $P_{\mathcal{F}} \in J_{X / B}^{d}(B)$. Soit $\mathfrak{U}_{X}(r ; \mathcal{F})$ le produit fibré sur $J_{X / B}^{d}$ de $P_{\mathcal{F}}$ et $\mathfrak{U}_{X}(r ; d)$, soient $p_{\mathcal{F}}: \mathfrak{U}_{X}(r ; \mathcal{F}) \rightarrow$ $\mathfrak{U}_{X}(r ; d)$ la deuxième projection et $\mathcal{E}_{\mathcal{F}}=p_{\mathcal{F}}^{*}(\mathcal{E})$.

Le schéma $g_{\mathcal{F}}: \mathfrak{U}_{X}(r ; \mathcal{F}) \rightarrow B$ paramétrise les faisceaux stables sur $X$ de rang $r$ et de déterminant isomorphe à $\mathcal{F}$ sur chaque fibre de $f$, et il est le schéma qu'on cherche.

Soient $T$ un $B$-schéma et $\mathcal{E} \in S_{X}(r ; \mathcal{F})(T)$. Le morphisme $\psi_{\mathcal{E}}: T \rightarrow$ $\mathfrak{U}_{X}(r ; d)$ se factorise à travers $\mathfrak{U}_{X}(r ; \mathcal{F})$. Et vice versa, pour tout point $Q: T \rightarrow \mathfrak{U}_{X}(r ; \mathcal{F})$, le fibré $(\mathrm{id} \times Q)^{*}\left(\mathcal{E}_{\mathcal{F}}\right)$ sur $X_{T}$ obtenu par le diagramme 
cartésien

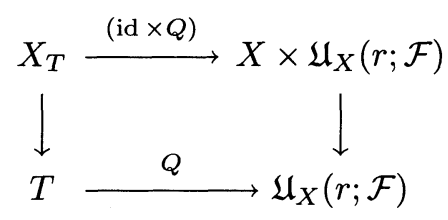

est dans $S_{X}(r ; \mathcal{F})$.

De plus, si $\mathcal{E} \in S_{X}(r ; \mathcal{F})(T)$, alors il existe $\mathcal{N} \in \operatorname{Pic}(T)$ tel que

$$
\left(\mathrm{id} \times \psi_{\mathcal{E}}\right)^{*}\left(\mathcal{E}_{\mathcal{F}}\right) \simeq \mathcal{E} \otimes \operatorname{pr}_{2}^{*}(\mathcal{N})
$$

On démontre les propriétés affirmées à l'aide des remarques suivantes.

On peut prouver que, si $Y$ est une courbe sur un corps et $\mathcal{F}$ un faisceau inversible sur $Y$ de degré $d$ (on rappelle que $r$ et $d$ sont premiers entre eux), alors $\mathfrak{U}_{X}(r ; \mathcal{F})$ est irréductible et lisse ( $c f$. [SD, chap. 1]). On peut donc prouver, de façon analogue à la démonstration du théorème 4.3, que $g_{\mathcal{F}}: \mathfrak{U}_{X}(r ; \mathcal{F}) \rightarrow B$ est un morphisme lisse et projectif de dimension rélative $\left(r^{2}-1\right)\left(g\left(X_{K}\right)-1\right)$.

Ce qui termine la démonstration.

Soit donc $f: X \rightarrow B$ une surface arithmétique (lisse); $\mathfrak{U}_{X}(r ; \mathcal{F})$ l'espace des fibrés $\mathcal{E}$ stables de rang $r$ et de déterminant $\mathcal{F}$ qui est de degré $d$ supposé assez grand; soit $\mathcal{E}_{\mathcal{F}}$ la famille universelle sur $X \times_{B} \mathfrak{U}_{X}(r ; \mathcal{F})$.

On construit maintenant une hauteur sur $\mathfrak{U}_{X}(r ; \mathcal{F})$ comme intersection d'Arakelov avec un faisceau inversible métrisé ample sur la fibre générique.

a) Rappels sur l'intégration des classes de Chern

Pour plus de détails sur cette partie, regarder [D], [E1], [E2] ou [GS2].

Soient $S$ un schéma (réduit) et $f: X \rightarrow S$ une famille lisse de courbes projectives de genre $g$; soit $\underline{\mathrm{Pic}}(S)$ la catégorie de Picard où les objets sont les fibrés inversibles sur $S$ et les morphismes les isomorphismes. On désigne par $\underline{\operatorname{Pic}}(X)$ la catégorie de Picard des fibrés inversibles sur $X$ et par $\underline{\operatorname{Vect}}(X)$ la catégorie des fibrés vectoriels sur $X$.

L'accouplement de Deligne est un foncteur

$$
\begin{aligned}
\operatorname{Pic}(X) \times \underline{\operatorname{Pic}}(X) & \longrightarrow \underline{\operatorname{Pic}}(S), \\
(\mathcal{L} ; \mathcal{M}) & \longrightarrow\langle\mathcal{L} ; \mathcal{M}\rangle_{D}
\end{aligned}
$$

qui commute avec tout changement de base, bilinéaire et si $\mathcal{L}=\mathcal{O}_{X}(D)$ avec $D$ diviseur relatif effectif, alors $\langle\mathcal{L} ; \mathcal{M}\rangle_{D}=N_{D / S}\left(\mathcal{M}_{\mid D}\right)$ où $N_{D / S}(\cdot)$ est la norme.

TOME $125-1997-\mathrm{N}^{\circ} 4$ 
Si $X$ et $S$ sont définies sur $\mathbb{C}$, si $S$ est lisse (donc $X$ aussi), et $\mathcal{L}$ et $\mathcal{M}$ sont métrisés sur $X$, alors on peut métriser de façon canonique $\langle\mathcal{L} ; \mathcal{M}\rangle_{D}$ et on a l'égalité entre formes différentielles sur $S$

$$
c_{1}\left(\langle\mathcal{L} ; \mathcal{M}\rangle_{D}\right)=\int_{X / S} c_{1}(\mathcal{L}) \wedge c_{1}(\mathcal{M})
$$

On peut intégrer la deuxième classe de Chern :

Il existe un foncteur

$$
\begin{aligned}
\underline{\operatorname{Vect}}(X) & \longrightarrow \underline{\operatorname{Pic}(S),} \\
\mathcal{E} & \longmapsto \mathrm{Ic}_{2}(\mathcal{E})
\end{aligned}
$$

tel que

a) $\operatorname{Ic}_{2}(\cdot)$ commute à tout changement de base;

b) si $\mathcal{L}$ est un fibré inversible sur $X$ alors $\operatorname{Ic}_{2}(\mathcal{L}) \simeq \mathcal{O}_{S}$ canoniquement;

c) si on a une suite exacte

$$
0 \rightarrow \mathcal{E}_{1} \longrightarrow \mathcal{E} \longrightarrow \mathcal{E}_{2} \rightarrow 0
$$

on a un isomorphisme canonique

$$
\operatorname{Ic}_{2}(\mathcal{E}) \simeq \operatorname{Ic}_{2}\left(\mathcal{E}_{1}\right) \otimes \operatorname{Ic}_{2}\left(\mathcal{E}_{2}\right) \otimes\left\langle c_{1}\left(\mathcal{E}_{1}\right) ; c_{1}\left(\mathcal{E}_{2}\right)\right\rangle_{D}
$$

De plus, si $X$ et $S$ sont définies sur $\mathbb{C}$ et si $S$ est lisse et $\mathcal{E}$ est muni d'une métrique $h$, on peut métriser de façon canonique $\operatorname{Ic}_{2}(\mathcal{E})$ et on a l'égalité entre formes différentielles sur $S$ :

$$
c_{1}\left(\operatorname{Ic}_{2}(\mathcal{E})\right)=\int_{X / S} c_{2}(\mathcal{E} ; h) .
$$

Fixons une métrique sur le faisceau dualisant relatif $\omega_{X / S}$, on peut alors écrire un théorème à la Riemann-Roch :

Proposition 6.2. - Fixons une métrique sur le fibré inversible $\operatorname{DetR} f_{*}\left(\mathcal{O}_{X}\right)$. Pour tout $\mathcal{E} \in \underline{\operatorname{Vect}}(X)$ métrisé, il existe une unique façon de métriser le fibré en droites $\operatorname{DetR} f_{*}(\mathcal{E})$ sur $S$, telle que les conditions suivantes soient vérifiées:

1) la métrique sur DetR $f_{*}\left(\mathcal{O}_{X}\right)$ est celle choisie;

2) (dualité de Serre) il existe une isométrie canonique

$$
\operatorname{DetR} f_{*}\left(\mathcal{E}^{*} \otimes \omega_{X / S}\right) \simeq \operatorname{DetR} f_{*}(\mathcal{E}) ;
$$

3) (théorème de Riemann-Roch) il existe une isométrie canonique

$\left(\operatorname{DetR} f_{*}(\mathcal{E})\right)^{2} \simeq\left\langle c_{1}(\mathcal{E}) ; c_{1}(\mathcal{E}) \otimes \omega_{X / S}^{-1}\right\rangle_{D} \otimes \operatorname{Ic}_{2}(\mathcal{E})^{-1} \otimes \operatorname{DetR} f_{*}\left(\mathcal{O}_{X}\right)^{2}$ 
Remarque. - Le mot «unique» signifie ici «qui est univoquement déterminé par $\mathcal{E}$ et par la métrique $\operatorname{sur} \mathcal{E}$.»

Preuve. - Du point de vue algébrique, les isomorphismes écrits sont canoniques, il faut simplement vérifier qu'ils soient des isométries. On peut utiliser 3) comme définition de la métrique cherchée, en imposant 1). La propriété 2) est alors évidente (et l'unicité aussi).

b) Hauteurs sur l'espace des modules des fibrés stables sur une courbe sur un corps de nombres

Soit $X_{K}$ une courbe lisse $\operatorname{sur} \operatorname{Spec}(K)$ ( $K$ corps des nombres); supposons que le modèle régulier minimal $X$ de $X_{K} \operatorname{sur} \operatorname{Spec}\left(\mathcal{O}_{K}\right)$ est à bonne réduction partout et que $X_{K}(K) \neq \emptyset$.

Soient $r$ et $d$ deux nombres entiers premiers entre eux ( $d$ assez grand), soit $\mathcal{F}_{K} \in \operatorname{Pic}\left(X_{K}\right)$ un fibré inversible de degré $d$; soit $\mathfrak{U}_{X_{K}}\left(r ; \mathcal{F}_{K}\right)$ l'espace des modules des fibrés stables de rang $r$ et de déterminant $\mathcal{F}_{K}$ sur $X_{K}$. On veut construire une hauteur sur la variété projective $\mathfrak{U}_{X_{K}}\left(r ; \mathcal{F}_{K}\right)$; donc, dans l'esprit de la philosophie d'Arakelov, on a besoin d'un modèle $\mathfrak{U}_{X}(r ; \mathcal{F})$ canonique de $\mathfrak{U}_{X_{K}}\left(r ; \mathcal{F}_{K}\right)$ sur $B$ et d'un fibré métrisé sur $\mathfrak{U}_{X}(r ; \mathcal{F})$. Soit $\mathcal{F}$ un modèle de $\mathcal{F}_{K}$ sur $X$.

Soit $\mathfrak{U}_{X}(r ; \mathcal{F})$ l'espace des modules construit au début de ce paragraphe; c'est un modèle sur $\mathcal{O}_{K}$ de $\mathfrak{U}_{X_{K}}\left(r ; \mathcal{F}_{K}\right)$.

Soit $K^{\prime}$ une extension finie de $K$ et soit $\mathcal{E}_{K^{\prime}}$ un fibré stable de déterminant $\mathcal{F}_{K^{\prime}}$ et rang $r$ sur $X_{K^{\prime}}$. Alors $\mathcal{E}_{K^{\prime}}$ définit un morphisme

$$
\operatorname{Spec}\left(K^{\prime}\right) \longrightarrow \mathfrak{U}_{X_{K}}\left(r ; \mathcal{F}_{K}\right) .
$$

Par le critère de propreté, il existe un unique morphisme

$$
\operatorname{Spec}\left(\mathcal{O}_{K^{\prime}}\right) \longrightarrow \mathfrak{U}_{X}(r ; \mathcal{F})
$$

qui prolonge le précédent; en utilisant la famille universelle sur l'espace $X \times{ }_{B} \mathfrak{U}_{X}(r ; \mathcal{F})$, on trouve un modèle «canonique» $\mathcal{E}$ du fibré $\mathcal{E}_{K^{\prime}}$ sur $\operatorname{Spec}\left(\mathcal{O}_{K^{\prime}}\right)$, stable sur chaque fibre : on munit $\mathcal{E}_{K^{\prime}}$ d'une métrique EinsteinHermite pour chaque place à l'infini de $K^{\prime}$. On dénote par $\widehat{c_{2}(\mathcal{E})}$ le nombre réel $\operatorname{deg}_{B^{\prime}}\left(\operatorname{Ic}_{2}(\mathcal{E})\right.$ ) (le degré est le degré d'Arakelov) et par $\left(\widehat{c_{1}(\mathcal{E})} ; \widehat{c_{1}(\mathcal{E})}\right)$ le nombre réel $\operatorname{deg}_{B^{\prime}}\left(\left\langle c_{1}(\mathcal{E}) ; c_{1}(\mathcal{E})\right\rangle_{D}\right)$ (intersection d'Arakelov).

Remarque. - L'égalité

$$
\left.2 r \widehat{c_{2}(\mathcal{E})}-(r-1)\left(\widehat{c_{1}(\mathcal{E})} ; \widehat{c_{1}(\mathcal{E})}\right)=\widehat{c_{2}(\operatorname{End}(\mathcal{E})}\right)
$$

implique que le nombre réel $2 r \widehat{c_{2}(\overline{\mathcal{E}})}-(r-1)\left(\widehat{c_{1}(\overline{\mathcal{E}})} ; \widehat{c_{1}(\overline{\mathcal{E}})}\right)$ ne varie pas si,

$$
\text { TOME } 125-1997-\mathrm{N}^{\circ} 4
$$


pour chaque $\sigma \in S_{\infty}$, on multiplie la métrique sur $\mathcal{E}_{\sigma}$ par une constante $e^{\alpha_{\sigma}} \in \mathbb{R}$. Puisque deux métriques d'Einstein-Hermite sur un fibré stable sont proportionnelles, le nombre $2 r \widehat{c_{2}(\mathcal{E})}-(r-1)\left(\widehat{c_{1}(\mathcal{E})} ; \widehat{c_{1}(\mathcal{E})}\right)$ ne dépend pas de la métrique d'Einstein-Hermite choisie.

Plus généralement, on verra au cours de la démonstration du théorème 6.3 que le nombre $h\left(\mathcal{E}_{K^{\prime}}\right)$ ne depend que du fibré $\mathcal{E}_{K^{\prime}}$ et pas des choix faits.

On définit alors

$$
h\left(\mathcal{E}_{K^{\prime}}\right)=\frac{1}{\left[K^{\prime}: \mathbb{Q}\right]}\left(2 r \widehat{c_{2}(\mathcal{E})}-(r-1)\left(\widehat{c_{1}(\mathcal{E})} ; \widehat{c_{1}(\mathcal{E})}\right)\right)
$$

le degré étant défini sur $\operatorname{Spec}\left(\mathcal{O}_{K^{\prime}}\right)$.

THÉORÈme 6.3. - Il existe un faisceau inversible ample $\Delta$ sur $\mathfrak{U}_{X_{K}}\left(r ; \mathcal{F}_{K}\right)$ tel que la fonction

$$
h(\cdot): \mathfrak{U}_{X_{K}}\left(r ; \mathcal{F}_{K}\right)(\bar{K}) \longrightarrow \mathbb{R}
$$

est une hauteur sur $\mathfrak{U}_{X_{K}}(r ; \mathcal{F})$ associée à $\Delta$.

Preuve. - Soit $\mathcal{E}_{\mathcal{F}}$ une famille universelle sur $X \times_{B} \mathfrak{U}_{X}(r ; \mathcal{F})$; soit $\left(\mathcal{E}_{\mathcal{F}}\right)_{K}$ sa restriction à la fibre générique. Le fibré

$$
\mathcal{L}=\left(\operatorname{DetR} p_{*}\left(\operatorname{End}\left(\left(\mathcal{E}_{\mathcal{F}}\right)_{K}\right)\right)\right)^{-1}
$$

inversible sur $\mathfrak{U}_{X_{K}}\left(r ; \mathcal{F}_{K}\right)$, où $p: X \times \mathfrak{U}_{X_{K}}\left(r ; \mathcal{F}_{K}\right) \rightarrow \mathfrak{U}_{X_{K}}\left(r ; \mathcal{F}_{K}\right)$ est la projection canonique, est ample.

En effet, $p_{*}\left(\operatorname{End}\left(\mathcal{E}_{\mathcal{F}}\right)\right)=\mathcal{O}_{\mathfrak{U}}$ et $R^{1} p_{*}\left(\operatorname{End}\left(\mathcal{E}_{\mathcal{F}}\right)\right)=T_{\mathfrak{U}}\left(T_{\mathfrak{U}}\right.$ étant le fibré tangent de $\mathfrak{U}_{X}(r ; \mathcal{F})$; d'où

$$
\operatorname{DetR} p_{*}\left(\operatorname{End}\left(\mathcal{E}_{\mathcal{F}}\right)\right)=\left(\operatorname{det}\left(T_{\mathfrak{U}}\right)\right)^{-1}=\omega_{\mathfrak{U}}
$$

$\omega_{\mathfrak{U}}$ étant le fibré canonique de $\mathfrak{U}_{X}(r ; \mathcal{F})$. On sait, d'après [DN, th. F], qu'on a un isomorphisme $\operatorname{Pic}\left(\mathfrak{U}_{X_{K}}\left(r ; \mathcal{F}_{K}\right)\right) \simeq \mathbb{Z}$ et que $\left(\omega_{\mathfrak{U}}\right)^{-1}$ est un diviseur effectif sur $\mathfrak{U}_{X_{K}}\left(r ; \mathcal{F}_{K}\right)$; donc $\left(\omega_{\mathfrak{U}}\right)^{-1}$ est ample, d'où l'amplitude $\operatorname{de}\left(\operatorname{DetR} p_{*}\left(\operatorname{End}\left(\mathcal{E}_{\mathcal{F}}\right)\right)\right)^{-1}$.

Pour tout $\sigma \in S_{\infty}$, on peut munir le fibré $\mathcal{E}_{\mathcal{F}_{\sigma}}$ sur $X_{\sigma} \times \mathfrak{U}_{X_{\sigma}}\left(r ; \mathcal{F}_{\sigma}\right)$ d'une métrique $h$ de classe $C^{\infty}$, de façon telle que pour tout $q \in \mathfrak{U}_{X_{\sigma}}\left(r ; \mathcal{F}_{\sigma}\right)$, le fibré stable $\mathcal{E}_{\mathcal{F}_{\sigma}}(q)=\mathcal{E}_{\mathcal{F}_{\sigma} \mid X_{\sigma} \times\{q\}}$ sur $X_{\sigma}$ muni de la métrique obtenue par restriction de la métrique $h$, soit un fibré métrisé d'Einstein-Hermite par rapport à $\mathrm{d} \mu_{\mathrm{Ar}}$ (proposition 5.2). 
On métrise de façon évidente le fibré inversible $\operatorname{DetR} p_{*}\left(\mathcal{O}_{X \times \mathfrak{U}_{X}(r ; \mathcal{F})}\right)$ $\operatorname{sur} \mathfrak{U}_{X}(r ; \mathcal{F})$.

Le fibré dualisant relatif $\omega_{X \times \mathfrak{U}_{X}(r ; \mathcal{F}) / \mathfrak{U}_{X}(r ; \mathcal{F})}$ est isomorphe au fibré $\operatorname{pr}_{1}^{*}\left(\omega_{X / \mathcal{O}_{K}}\right)$; donc il est métrisé canoniquement à l'aide de la métrique d'Arakelov (permise) sur $\omega_{X / \mathcal{O}_{K}}$.

La proposition 6.2 affirme, donc, que le fibré sur $\mathfrak{U}_{X}(r ; \mathcal{F})$, ample sur la fibre générique, $\Delta=\left(\operatorname{DetR} p_{*}\left(\operatorname{End}\left(\mathcal{E}_{\mathcal{F}}\right)\right)\right)^{\otimes-2}$ est métrisé de façon canonique.

On remarque que, puisque $\operatorname{End}\left(\mathcal{E}_{\mathcal{F}}\right)=\mathcal{E}_{\mathcal{F}} \otimes \mathcal{E}_{\mathcal{F}}^{*}$, on a des isométries canoniques :

- $\left\langle c_{1}\left(\operatorname{End}\left(\mathcal{E}_{\mathcal{F}}\right)\right) ; c_{1}\left(\operatorname{End}\left(\mathcal{E}_{\mathcal{F}}\right)\right)\right\rangle_{D} \simeq \mathcal{O}_{\mathfrak{U}_{X}(r ; \mathcal{F})}\left(\mathcal{O}_{\mathfrak{U}_{X}(r ; \mathcal{F})}\right.$ est muni de la métrique triviale);

- $\operatorname{Ic}_{2}\left(\operatorname{End}\left(\mathcal{E}_{\mathcal{F}}\right)\right) \simeq\left(\operatorname{Ic}_{2}\left(\mathcal{E}_{\mathcal{F}}\right)\right)^{\otimes 2 r} \otimes\left(\left\langle c_{1}\left(\mathcal{E}_{\mathcal{F}}\right) ; c_{1}\left(\mathcal{E}_{\mathcal{F}}\right)\right\rangle_{D}\right)^{\otimes-(r-1)}$.

Donc, toujours pour la proposition 6.2 , on a une isométrie canonique

$$
\begin{aligned}
& \Delta \simeq\left(\mathrm{Ic}_{2}\left(\mathcal{E}_{\mathcal{F}}\right)\right)^{\otimes 2 r} \otimes\left(\left\langle c_{1}\left(\mathcal{E}_{\mathcal{F}}\right) ; c_{1}\left(\mathcal{E}_{\mathcal{F}}\right)\right\rangle_{D}\right)^{\otimes-(r-1)} \\
& \otimes\left(\operatorname{DetR} \pi_{*}\left(\mathcal{O}_{X \times \mathfrak{U}_{X}(r ; \mathcal{F})}\right)\right)^{\otimes-2} .
\end{aligned}
$$

Soient $K^{\prime}$ une extension (finie) de $K, \mathcal{O}_{K^{\prime}}$ son anneau d'entiers; on dénote $B^{\prime}=\operatorname{Spec}\left(\mathcal{O}_{K^{\prime}}\right)$ et $X^{\prime}=X \times_{B} B^{\prime}$. Soit maintenant $\mathcal{E}$ appartenant à $S_{X}(r ; d)\left(B^{\prime}\right)$; pour tout $\sigma \in S_{\infty}$, on munit $\mathcal{E}_{\sigma}$ d'une métrique d'EinsteinHermite par rapport à la métrique d'Arakelov sur $X_{\sigma}$; on peut associer à $\mathcal{E}$ un morphisme

$$
\psi_{\mathcal{E}}: B^{\prime} \longrightarrow \mathfrak{U}_{X}(r ; \mathcal{F})
$$

et donc on a un diagramme commutatif

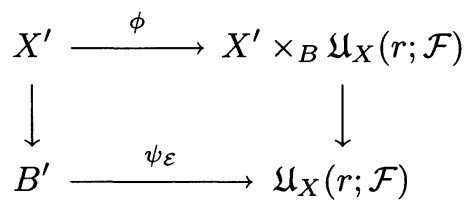

et il existe $\mathcal{M} \in \operatorname{Pic}\left(B^{\prime}\right)$ tel que $\phi^{*}\left(\mathcal{E}_{\mathcal{F}}\right) \simeq \mathcal{E} \otimes f^{*}(\mathcal{M})$; d'où

$$
\phi^{*}\left(\operatorname{End}\left(\mathcal{E}_{\mathcal{F}}\right)\right) \simeq \operatorname{End}(\mathcal{E})
$$

et, par fonctorialité :

$$
\psi_{\mathcal{E}}^{*}(\Delta) \simeq\left(\mathrm{Ic}_{2}(\mathcal{E})\right)^{\otimes 2 r} \otimes\left(\left\langle c_{1}(\mathcal{E}) ; c_{1}(\mathcal{E})\right\rangle_{D}\right)^{\otimes-(r-1)} \otimes\left(\operatorname{DetR} f_{*}\left(\mathcal{O}_{X}\right)\right)^{\otimes-2}
$$

TOME $125-1997-\mathrm{N}^{\circ} 4$ 
D'où on $\mathrm{a}$ :

$$
\begin{aligned}
h_{\Delta}\left(\psi_{\mathcal{E}}\left(B^{\prime}\right)\right) & =\frac{\operatorname{deg} \psi(\mathcal{E})^{*}(\Delta)}{\left[K^{\prime}: \mathbb{Q}\right]} \\
& =\frac{1}{\left[K^{\prime}: \mathbb{Q}\right]}\left(2 r \widehat{c_{2}(\mathcal{E})}-(r-1)\left(\widehat{c_{1}(\mathcal{E})} ; \widehat{c_{1}(\mathcal{E})}\right)+C\right.
\end{aligned}
$$

où $C$ est une constante qui ne dépend pas de $\mathcal{E}$.

Ce qui achève la démonstration du théorème.

REMARQUES.

a) On a vu, dans la démonstration du théorème 6.3 que

$$
\Delta=\operatorname{DetR} p_{2 *}(\operatorname{End}(\mathcal{E}))^{\otimes-2}=c_{1}\left(T_{\mathfrak{U}}\right)^{\otimes 2}
$$

$T_{\mathfrak{U}}$ étant le fibré tangent à $\mathfrak{U}_{X}(d ; \mathcal{F})$.

b) On peut aussi expiciter la constante $C$ dans (2) :

$$
\left.C=-2 \widehat{\operatorname{deg}}\left(\operatorname{DetR} f_{*}\left(\mathcal{O}_{X}\right)\right)\right) .
$$

La constante $C$ peut être explicitée en utilisant la formule de FaltingsNoether $(c f .[\mathrm{F}])$.

Corollaire 6.4. - Il existe une constante $M$ telle que, pour tout fibré $\mathcal{E} \in S_{X_{\bar{K}}}\left(r ; \mathcal{F}_{K}\right)$ métrisé, on ait $h(\mathcal{E}) \geq M$.

Remarque. - En effet, on peut faire la même construction pour $\mathfrak{U}_{X}(r ; d)$ et on aura construit une hauteur sur ce schéma; malheureusement $\operatorname{DetR} p_{2 *}(\operatorname{End}(\mathcal{E}))$ n'est pas ample, en général.

Remarque. - D'après $[\mathrm{Mo}]$ ou [So], on a toujours $h\left(\mathcal{E}_{K}\right) \geq 0$. Plus généralement, ils prouvent que si $\mathcal{E}$ est un fibré sur une surface arithmétique (quelconque) semi-stable sur la fibre générique de $X$, alors

$$
2 r \widehat{c_{2}(\mathcal{E})}-(r-1)\left(\widehat{c_{1}(\mathcal{E})} ; \widehat{c_{1}(\mathcal{E})}\right) \geq 0 .
$$

Donc la hauteur qu'on a contruite est toujours non négative.

Comme corollaire du théorème 6.3 on trouve :

THÉORÈmE 6.5. - Soient $f: X \rightarrow \operatorname{Spec}\left(\mathcal{O}_{K}\right)$ une surface arithmétique lisse et $r$ et $d$ deux nombres entiers premiers entre eux. Soit $\mathcal{F} \in \operatorname{Pic}(X)$ un fibré inversible de degré $d$ (sur la fibre générique). Soit $S_{X}(r ; \mathcal{F})$ l'ensemble des fibrés stables de déterminant isomorphe à $\mathcal{F}$ sur chaque 
fibre de $f$ et de rang $r$ sur $X$. Supposons que pour tout $\sigma \in S_{\infty}$ et pour tout $\mathcal{E} \in S_{X}(r ; \mathcal{F})$, on a fixé une métrique de Einstein-Hermite sur $\mathcal{E}_{\sigma}$ par rapport à la métrique d'Arakelov sur $X_{\sigma}$. Soit $A$ un nombre réel; alors il n'existe qu'un nombre fini de fibrés $\mathcal{E} \in S_{X}(r ; \mathcal{F})$ tels que

$$
h(\mathcal{E})=\frac{1}{[K: \mathbb{Q}]}\left(2 r \widehat{c_{2}(\mathcal{E})}-(r-1)\left(\widehat{c_{1}(\mathcal{E})} ; \widehat{c_{1}(\mathcal{E})}\right)\right) \leq A
$$

La seule remarque à faire est que $\operatorname{Pic}(B)$ est fini!

\section{BIBLIOGRAPHIE}

[A] Arakelov (S.Ju.). - Intersection Theory of Divisors on an Arithmetic Surface, Math. U.S.S.R. Isvestija, t. 8, 6, 1974.

[BLR] Bosch (S.), Lutkebohmert (W.), Raynaud (M.). - Néron Models, Ergebnisse der Mathematik, t. 3, Bd. 21, Springer-Verlag, Berlin, Heidelberg, New York, 1990.

[D] Deligne (P.). - Le déterminant de la cohomologie, Contemporary Mathematics, t. 67, 1987.

[DN] Drezet (J.-M.), Narasimhan (M.S.). - Groupe de Picard des variétés de modules de fibrés semi-stables sur les courbes algébriques, Invent. Math., t. 97, 1989.

[Do] Donaldson (S.K.). - A New Proof of a Theorem of Narasimhan and Seshadri, J. Diff. Geometry, t. 18, 1983.

[F] Faltings (G.). - Calculus on Arithmetic Surfaces, Ann. of Maths, t. 119, 1984 .

[E] Ецкік (R.). - Fibrés d'intersection et intégrales de classes de Chern, Ann. Sci. École Norm. Sup., $4^{\mathrm{e}}$ série, t. 22, 1989.

[E2] ElkiK (R.). - Métriques sur les fibrés d'intersection, Duke Math. J., t. 61, $\mathrm{n}^{\mathrm{o}} 1,1989$.

[Ga] Gasbarri (C.). - Accouplement de Deligne et hauteurs de NéronTate, C. R. Acad. sci. Paris, t. 323, série I, 1996.

[GS] Gillet (H.), Soulé (C.). - Arithmetic Intersection Theory, Publications Math. IHES, t. 72, 1990.

[GS2] Gillet (H.), Soulé (C.). - Characteristic Classes for Algebraic Vector Bundles with Hermitian Metrics I, II, Ann. Math., t. 131, 1990.

TOME $125-1997-\mathrm{N}^{\circ} 4$ 
[G] Grothendieck (A.). - Fondements de la géométrie algébrique, Séminaire Bourbaki 1957-1962, Secrétariat de Math., Institut Henri Poincaré, 1962.

[LP] Le Potiers (J.). - Fibrés vectoriels sur les courbes algébriques, notes polycopiées, Université Paris 7, 1991.

[LP2] Le Potiers (J.). - Espaces de modules de faisceaux semi-stables sur le plan projectif, preprint, Université Paris 7, 1992.

[Ma] Maruyama (M.). - Moduli of Stable Sheaves, J. Math. Kyoto University, I, t. 17, 1977; II, t. 18, 1978.

[MB] Moret-Bailly (L.). - Métriques permises, Séminaire sur les pinceaux arithmétiques : la conjecture de Mordell (L. Szpiro, éd.), exposé 2, Astérisque 127, Paris, 1985.

[MB2] Moret-Bailly (L.). - La formule de Noether pour les surfaces arithmétiques, Inv. Math., t. 98, 1989 .

[Mu] Mumford, (D.). - Lectures on Curves on Algebraic Surfaces, Annals Maths Studies, t. 59, Princeton, 1966.

[GIT] Mumford (D.), Fogarty (J.). - Geometric Invariant Theory. Springer-Verlag, 2nd ed., 1982.

[Ko] Kobayashi (S.). - Differential Geometry of Complex Vector Bundles, Kanô Memorial, lecture 5, Iwahami Shoten and Princeton University Press, 1987 .

[Mi] Milnor (J.). - Introduction to Algebraic K-Theory, Annals Maths. Studies, t. 72, Princeton Univ. Press., 1971.

[Mo] Moriwaki (A.). - Inequalities of Bogomolov-Gieseker's Type on Arithmetic Surfaces, Duke Math. J., t. 74, 1994.

[NS] Narasimhan (M.), Ramanam (S.). - Deformations of moduli space of vector bundles over an algebraic curve, Ann. of Math., t. 101, 1975 .

[R] Ramanam (S.). - The Moduli Space of Vector Bundles on a Algebraic Curve, Math. Ann., t. 200, 1973.

[S] Seshadri (C.S.). - Geometric Reductivity over Arbitrary Base, Advances in Math, t. 26, 1977.

[SD] SEShAdRi (C.S.). - Fibrés vectoriels sur les courbes algébriques, Astérisque, t. 96, 1982.

[So] Soulé (C.). - A Vanishing Theorem on Arithmetic Surfaces, Invent. Math., t. 116, 1994.

[Sz] SzPIRo (L.). - Degrés, intersections, hauteurs, Séminaire sur les pinceaux arithmétiques : la conjecture de Mordell (L. Szpiro éd.), exposé 1, Astérisque, t. 127, Paris, 1985. 